\title{
Mathematical modeling of collagen turnover in biological tissue
}

\author{
Pablo Sáez • Estefanía Peña • Miguel \\ Ángel Martínez • Ellen Kuhl
}

Received: date / Accepted: date

\begin{abstract}
We present a theoretical and computational model for collagen turnover in soft biological tissues. Driven by alterations in the mechanical environment, collagen fiber bundles may undergo important chronic changes, characterized primarily by alterations in collagen synthesis and degradation rates. In particular, hypertension triggers an increase in tropocollagen synthesis and a decrease in collagen degradation, which lead to the well-documented overall increase in collagen content. These changes are the result of a cascade of events, initiated mainly by the endothelial and smooth muscle cells. Here, we represent these events collectively in terms of two internal variables, the concentration of growth factor TGF- $\beta$ and tissue inhibitors of metalloproteinases TIMP. While the upregulation of the former increases the collagen density, the upregulation of the latter increases the collagen density through decreasing matrix metalloproteinase MMP. We establish a mathematical theory for mechanically-induced collagen turnover and introduce a computational algorithm for its robust and efficient solution. We demonstrate that our model can accurately predict the experimentally observed collagen increase in response to hypertension reported in literature. Ultimately, the model can serve as a valuable tool to predict the chronic adaptation of collagen content to restore the homeostatic equilibrium state in vessels with arbitrary micro-structure and geometry.
\end{abstract}

\footnotetext{
Pablo Sáez

Department of Mechanical Engineering, Stanford University, Stanford, CA 94305, USA

Applied Mechanics and Bioengineering. Aragón Institute of Engineering Research (I3A),

University of Zaragoza, Zaragoza, Spain Tel.: +34-976-760000

E-mail: psaez@unizar.es

Estefanía Peña $\cdot$ Miguel Ángel Martínez

Applied Mechanics and Bioengineering. Aragón Institute of Engineering Research (I3A), University of Zaragoza, Zaragoza, Spain

CIBER-BBN. Centro de Investigación Biomédica en Red en Bioingeniería, Biomateriales y Nanomedicina, Spain

Ellen Kuhl Department of Mechanical Engineering, Bioengineering and Cardiothoracic Surgery, Stanford University, Stanford, CA 94305 USA
} 
Keywords Collagen, turnover, growth, remodeling, hypertension, blood vessels

\section{Motivation}

One of the most common forms of vascular disease is hypertension, a chronic medical condition in which the blood pressure is elevated (Freis, 1960). We can distinguish between two main types of hypertension, essential and secondary. Essential hypertension (Folkow, 1982; Carretero \& Oparil, 2000) is the most common type. It is not associated with any particular causes, but it is thought that lifestyle and genetics can play an important role in its pathogenesis. Secondary hypertension is associated with an underlying disease of the endocrine system.

It is widely accepted that stimuli of endothelial cells play an important role in the regulation of vascular adaptation (Wu \& Bohr, 1990; Hecker et al., 1993; Masaki, 1993; Rubanyi \& Polokoff, 1994; Li et al., 2005). In addition, smooth muscle cells (SMC) may trigger a mechanochemical regulation of the vascular structure (Folkow, 1949; Kuo et al., 1990; Bevan \& Laher, 1991). In both cases, the elevated blood pressure causes increased mechanical stresses on the vascular structure, manifesting themselves in changes of the shear stresses and increased circumferential stresses in the vascular wall. These stimuli initiate two fundamentally different acute and chronic phenomena.

SMCs produce an acute contractile force in a short period of time due to changes in blood pressure. This corresponds to the so-called myogenic tone (Brayden \& Nelson, 1992; Schubert \& Mulvany, 1999; Henrion, 2005), which is directly related to the intracellular calcium concentration and to the ion channels of the cell (Adams et al., 1989; Brayden \& Nelson, 1992; Davis \& Hill, 1999). This force attempts to compensate the over-stress in the wall in order to maintain the same vessel diameter, see, e.g., Osol (1995); Haga et al. (2007) for a review of the molecular and biochemical aspects of SMC.

SMCs start to grow chronically, via hypertrophy, hyperplasia or both (Owens et al., 1981; Owens, 1989), to restore the homeostatic stress state, also known as the basal tone. This chronic adaptation leads to the well-documented thickening of the vessel wall. These changes are more pronounced in small or resistance vessels (Folkow et al., 1958; Mulvany \& Aalkjaer, 1990). Bishop \& Lindahl (1999) showed that the increase in extracellular matrix (ECM) deposition can be caused by hyperplasia or by the increase in cell synthesis, while Owens et al. (1981) identified hypertrophy to be the main origin of SMC growth. During these SMC adaptations, there are important changes in the ECM, not only in the rate of deposition, but also in the rate of degradation of collagen. These two processes disturb the natural deposition-degradation balance of collagen in the healthy vessel. This perturbation of the equilibrium state can cause serious diseases or conditions, such as fibrosis in the peripheral vasculature and the heart, aneurysm formation or vessel stiffening.

The biological regulation of hypertension has been widely studied over the past decades. Focusing on the collagen turnover of the vasculature, there is general agreement that collagen changes are mediated by an increase in collagen deposition and changes in collagen degradation, which perturb the biological 
equilibrium state. Both deposition and degradation can occur at the intracellular (collagen molecules) or extracellular (tropocollagen) levels. There have been several in-vitro and in-vivo experiments studying these processes in detail. It is generally agreed that the ground substance plays a fundamental role in this turnover regulation.

In terms of deposition, fibrogenic cytokine proteins such as the transforming growth factor TGF- $\beta$ are the most important regulators (see e.g. Border \& Noble (1994); Wrana et al. (1994) and references therein), which also play a prominent role in SMC proliferation (Owens, 1995; Raines, 2004). Bishop (1998) showed increased rates of procollagen deposition accompanied by an increase in the proliferation of SMC. Since SMCs and fibroblasts are attached to the extracellular matrix, they are capable of sensing lower strains in stiffer matrices modifying, therefore, its active behavior. Butt et al. (1995) reported that SMC release the TGF- $\beta$ growth factor associated with an increase in procollagen deposition and a decrease in collagen degradation. They also showed that both SMC and endothelial cells are the source of the important platelet-derived growth factor PDGF.

In terms of degradation, Metalloproteinase enzymes (MMP) and tissue inhibitors of metalloproteinase (TIMP), in particular their aspect ratio MMP/TIMP, may be the most important metric for quantifying the evolution of degradation. MMP (Galis et al., 1994; Galis \& Khatri, 2002), in addition to other regulatory mechanisms such as differentiation and apoptosis of cells, are responsible for extracellular matrix degradation in general, and for collagen degradation in particular. TIMPs are a type of inhibitor of metalloproteinase. In hypertension, TIMP have been reported to increase, decreasing the total MMP, which, in turn, decreases the rate of collagen degradation. O'Callaghan \& Williams (2000) have shown that the amount of collagen turnover increases with the magnitude of the strain imposed on SMC in in-vitro experiments. They also reported the production of MMP-2, a gelatinase-degrading enzyme and TGF- $\beta$ (see also Sarzani et al. (1989); Hamet et al. (1991)), which could be stimulated by cyclic stretching. This acts as an important regulator of ECM production, mainly by inhibition of MMP-1 and by increasing the activity of MMP-2. Other studies also suggest that mechanical loading increases the proliferation of endothelial cells and the production of endothelins ET-1 (Masaki et al., 1991; Masaki, 1993; Li et al., 2005), which is involved in the constriction of blood vessels.

Structurally, collagen is an important protein found in many connective and fibrous tissues. The extracellular collagen molecule, procollagen, is made up of three left-handed helix polypeptides, the so-called $\alpha$-chains, coiled-up in a right-handed helical structure, about $300 \mathrm{~nm}$ long and $1.5 \mathrm{~nm}$ in diameter (Bella et al., 1994; Orgel et al., 2001; Bhattacharjee \& Bansal, 2005). Collagen molecules assemble along a given direction through covalent bonds to form collagen microfibrils, constituting the basic building block of collagen fibrils (Baselt et al., 1993; Hulmes et al., 1995; Orgel et al., 2006, 2011). The collagen fibrils are gathered in the extracellular matrix to form bundles of collagen fibers. Fig. 1 shows a representation of such a structure.

Collagen bears the major part of the load transmitted through the tissue, and substantial research has been devoted to understanding its hierarchical mi- 
crostructure at and across the different scales, see (Fratzl, 2008) for a review, as in bones (Fratzl et al., 2004), bundles of collagen (Alastrue et al., 2010), collagen fibrils (van der Rijt et al., 2006; Shen et al., 2008), microfibrils (Gautieri et al., 2011) and tropocollagen molecules (Buehler \& Wong, 2007), where the latter two used molecular dynamics analysis.

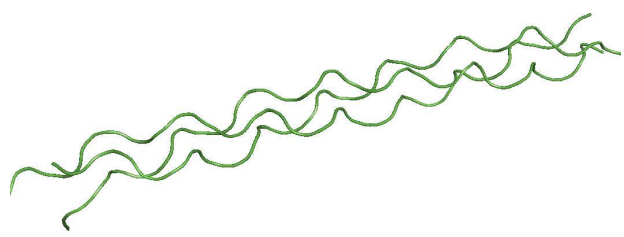

(a) Tropocollagen molecule

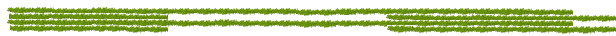

(b) Microfibril representation

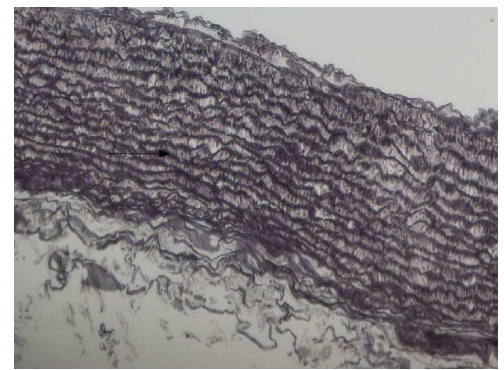

(c) Histology showing the bundles of collagen fibers

Fig. 1 Hierarchical structure of collagen in a blood vessel wall.

There is ample evidence that collagen turnover changes in response to hypertension. These deposition-degradation changes implicitly imply the growth of the tissue or, to be more precise, changes in volume, density or both. There have been different approaches to the computational modeling of growth and adaptation has seen different approaches in the context of biological tissue, see, e.g, Taber (1995); van der Meulen \& Prendergast (2000); Kelly \& Prendergast (2005); Ambrosi et al. (2011); Jones \& Chapman (2012). One fundamental difference, when compared to non-living materials, is related to the open and closed system approximation, see, e.g., Kuhl \& Steinmann (2003).

Existing models can be classified into volumetric growth and density growth models (Himpel et al., 2005). The first allow changes in volume, while keeping the density constant (Kuhl et al., 2007). The second keep the volume constant, while the density changes. Pang et al. (2011) presented these concepts for isotropic growth and Menzel $(2004,2007)$ for anisotropic growth. The kinematics of these approximations were first described by Skalak et al. (1982) and Rodriguez et al. (1994).

An alternative theoretical and computational approach is presented in Humphrey \& Rajagopal (2002, 2003), based on the constrained mixture theory, where 
several constituents of a tissue are allowed to grow independently. Gleason \& Humphrey (2004, 2005); Klisch et al. (2005) follow this approach for constrained mixtures and Ateshian (2007) for reactive mixtures. A key contribution is the work of Fung \& Liu (1989), amongst many others, which demonstrates that the volumetric growth of blood vessels induces a change in the natural configuration of the tissue, and induces residual stresses. Diffusion and cells migration models (Javierre et al., 2009; Cumming et al., 2010; Boyle et al., 2011) are other interesting approaches to the problem of biological tissue adaptation.

In this contribution, we present a theoretical and computational model for the adaptation of collagen in response to hypertension, or, more generally, to arbitrary changes in blood pressure. Given a history of SMC evolution and acute contraction, we characterize the deposition-degradation behavior of collagen. In this model, mechanical stimuli within the vascular wall are the major driving force for the chemical and mechanical adaptation. We assume ECM is the first source of production of TGF- $\beta$ and TIMP where the latter changes the turnover of MMP and together with the TGF- $\beta$ causes the collagen to evolve to non-physiological situations. Our model takes into account the deposition and degradation of collagen fibrils due to changes of the linking and demoting rates of the tropocollagen molecules (Fields, 1991; Bode et al., 1999; Chung et al., 2004). The density changes of the fibril are computed numerically by means of an incremental iterative Newton-Raphson scheme. Structurally, collagen fibers are represented using an anisotropic microsphere concept, which has recently been used to model soft fibrous structures (Miehe et al., 2004; Alastrué et al., 2009). We account for the anisotropy of the vessel by means of a Bingham statistical distribution function. Collagen fibrils are modeled using the worm-like chain model.

As illustrated in Fig. 2, we adopt a simplified scheme for the cascade of events initiated by hypertension. In short, the model captures the mechanical stimuli of the SMCs which start the production of TGF- $\beta$ and TIMP, which in turn regulate MMP. Note that the regulation of these substances depends on all the passive behavior of the tissue: that is, SMC, collagen and elastin. Based on the production of these substances, collagen turnover is either up regulated or downregulated.

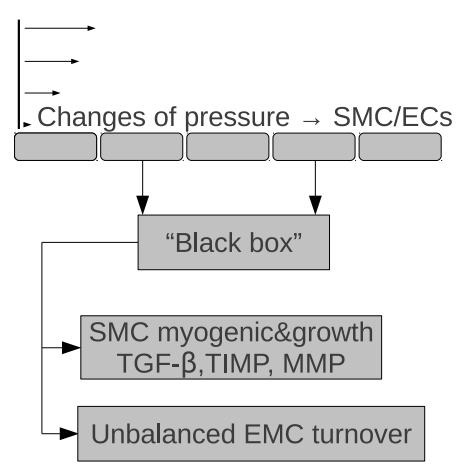

Fig. 2 Simplified assumption of the underlying process in hypertension. 
This manuscript is organized as follows. First, we present the passive model of the vessel wall with a particular focus on mid-size arteries. We begin by giving a brief description of the kinematic problem and the hyperelastic framework which characterizes the behavior of SMC and collagen. In order to provide an accurate description of the underlying structure, we adopt an anisotropic approach for both. Next, we introduce the underlying thermodynamics and stress expressions. Then, we expand the formulation to incorporate the adaptive response and characterize substance turnover. In particular, we focus on the synthesis of TGF- $\beta$ and TIMP and the absorption of MMP. Based on these changes, we model the dynamic turnover of collagen. We then discuss the computational treatment of the governing equations. To illustrate the features of the proposed model, we explore the model problem of hypertension. We conclude with a brief summary and discussion.

\section{Methods. Passive behavior.}

\subsection{Kinematics}

We apply the classical non-linear kinematic setting of continuum mechanics, see, e.g, Marsden \& Hughes (1994). Let $\mathbf{X} \in \Omega_{0}$ be a particle in the reference configuration and $\mathbf{x} \in \Omega$ the same particle in the spatial configuration $\Omega$. The mapping $\varphi$ relates both configurations, the deformation gradient $\mathbf{F}$ relates the corresponding tangent spaces, as shown in Fig. 3, and its Jacobian $J$ relates the corresponding volumes,

$$
\mathbf{F}=\nabla_{\mathbf{X}} \boldsymbol{\varphi} \quad \text { and } \quad J=\operatorname{det} \mathbf{F} .
$$

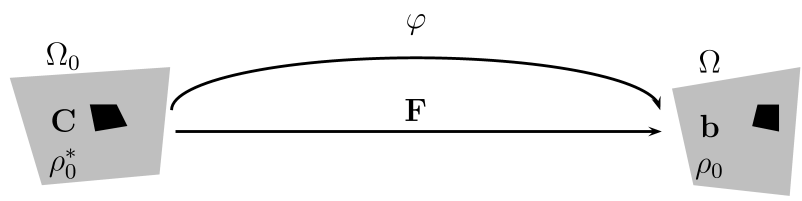

Fig. 3 Visualization of kinematics from the reference to the spatial configuration.

Given these definitions, we can introduce the right Cauchy-Green tensor $\mathbf{C}$ and the stretch $\lambda$ along the direction $\mathbf{n}$,

$$
\lambda=\left[\mathbf{n} \cdot \mathbf{F}^{t} \cdot \mathbf{F} \cdot \mathbf{n}\right]^{1 / 2}=[\mathbf{n} \cdot \mathbf{C} \cdot \mathbf{n}]^{1 / 2} \quad \text { with } \quad \mathbf{C}=\mathbf{F}^{t} \cdot \mathbf{F} .
$$

The volumetric-deviatoric decomposition can be parameterized through the multiplicative split of the deformation gradient and the right Cauchy-Green tensor as

$$
\mathbf{F}=J^{1 / 3} \mathbf{I} \cdot \overline{\mathbf{F}} \quad \text { and } \quad \mathbf{C}=J^{2 / 3} \mathbf{I} \cdot \overline{\mathbf{C}}
$$

respectively, where $\mathbf{I}$ denotes the second order identity tensor. The first terms of both expressions represent the volumetric parts and the second terms, $\overline{\mathbf{F}}$ and 
$\overline{\mathbf{C}}$, represent the deviatoric parts. Based on these definitions, we can introduce the modified invariants

$$
\bar{I}_{1}=\operatorname{tr}\left(\overline{\mathbf{F}}^{t} \cdot \overline{\mathbf{F}}\right) \quad \text { and } \quad \bar{I}_{4}=\mathbf{n} \cdot \overline{\mathbf{F}}^{t} \cdot \overline{\mathbf{F}} \cdot \mathbf{n}=\bar{\lambda}^{2} .
$$

\subsection{Hyperelastic framework}

The hyperelastic formulation is a common mathematical description in the modeling of soft tissue, mainly because of its highly non-linear and quasiincompressible behavior (Famaey \& Sloten, 2008). The incompressible character is due to the high water content of soft biological tissues and particularly in blood vessels. The use of a hyperelastic formulation relies on the definition of a Helmholtz strain energy density function (SEDF) from which we can derive stress, constitutive tangents, and dissipation using the framework of thermodynamics. For the study of soft tissue in general, and blood vessels in particular, the additive decomposition of the SEDF described first by Flory (1961) can be written as

$$
\begin{aligned}
& \Psi=\Psi_{\mathrm{vol}}+\Psi_{\mathrm{dev}}=\Psi_{\mathrm{vol}}(J)+\Psi_{\mathrm{iso}}\left(\bar{I}_{1}\right)+\Psi_{\mathrm{ani}}\left(\bar{I}_{4}\right) \text { with } \\
& \Psi_{\mathrm{ani}}\left(\bar{I}_{4}\right)=\Psi_{\mathrm{smc}}\left(\bar{I}_{4}\right)+\Psi_{\mathrm{col}}\left(\bar{I}_{4}, \rho_{0}\right) .
\end{aligned}
$$

The first term, $\Psi_{\mathrm{vol}}$, that characterizes the volumetric part of the energy, can be used to impose quasi-incompressibility of soft tissues. The remaining term, $\Psi_{\mathrm{dev}}$, characterizes the deviatoric or isochoric part of the SEDF, which is related to the SMC, elastin, and collagen contents while their water content has already been taken into account in $\Psi_{\text {vol }}$. It can be further decomposed into an isotropic part, $\Psi_{\text {iso }}$, associated to the elastin in the vessel wall, which we consider to remain constant throughout this work. The last term, $\Psi_{\text {ani }}$, is related to the anisotropy of the vessel attributed to the SMC, $\Psi_{\mathrm{smc}}$, and the collagen fibers, $\Psi_{\mathrm{col}}$, where the latter depends on the local density $\rho_{0}$ of the collagen. These two constituents (SMCs and collagen) play an important role in hypertensive disease, and are therefore the ones to be studied in this contribution. Blood vessels are highly directional non-linear structures and it is critical to utilize anisotropic models to accurately capture their constitutive behavior.

\subsection{Smooth muscle cell behavior}

SMCs play an important role both in the regulatory mechanism of the ECM and in the contraction of vessel walls. The latter, the active behavior, also known as basal tone, allows the vessel to contract in order to maintain the same stress state when subject to elevated pressure levels. The active behavior is controlled primarily by the calcium concentration regulated by the ion channel opening and closing. Recent mathematical models by Zulliger et al. (2004); Murtada et al. (2010); Famaey et al. (2011) describe this active behavior in more detail. Here, we will adopt the first of these three approaches, which provides a sufficiently accurate representation of the SMC behavior, whereas the 
latter two models characterize calcium dynamics in more detail. The behavior of the SMC is defined by a SEDF as follows,

$$
\Psi_{\mathrm{smc}}\left(\bar{I}_{4}\right)= \begin{cases}0, & \text { if } \bar{I}_{4}<1 \\ \mathrm{~S}_{1} \cdot \mu_{\mathrm{smc}}\left[\bar{I}_{4}-1\right] & \text { if } \bar{I}_{4} \geq 1\end{cases}
$$

where $\mu_{\mathrm{smc}}$ is an elastic constant. $S_{1}$ is a dimensionless function that sets the level of contraction as

$$
S_{1}\left(\bar{I}_{4}\right)=\mathrm{S}_{\mathrm{bsl}}+\left[1-\mathrm{S}_{\mathrm{bsl}}\right] \frac{1}{2}\left[1+\operatorname{erf}\left(\frac{\overline{I_{4}}-\mu}{\sqrt{2} \sigma}\right)\right]
$$

ranging from a basal or homeostatic value $\left(\mathrm{S}_{\mathrm{bsl}}\right)$ to the fully contracted state, characterized through a sigmoid-type function. The Gaussian distribution used to described the activation of the SMCs is characterized by the critical engagement deformation $\mu$ and its half-width $\sigma$ with an error function err. Fig. 4 displays the sensitivity analysis of the SMC stress response for varying sets of material parameter values.

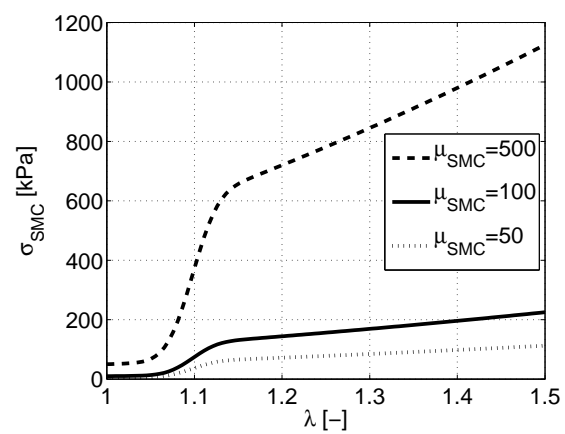

(a) Variation of $\mu_{S M C}$

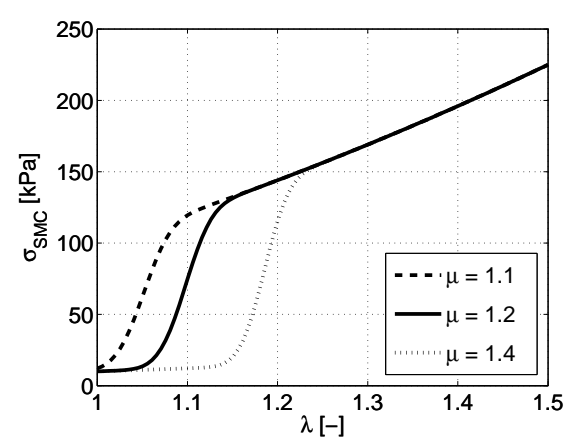

(c) Variation of $\mu$

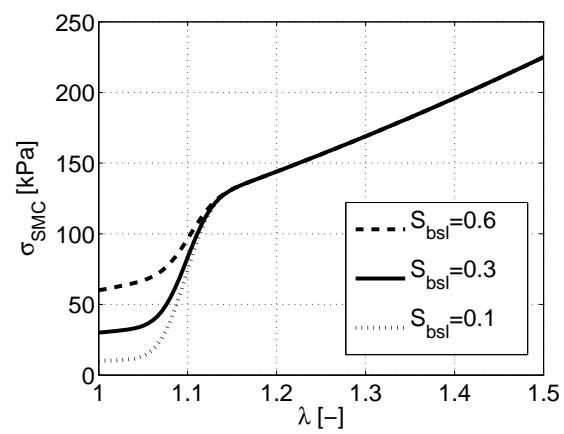

(b) Variation of $S_{b s l}$

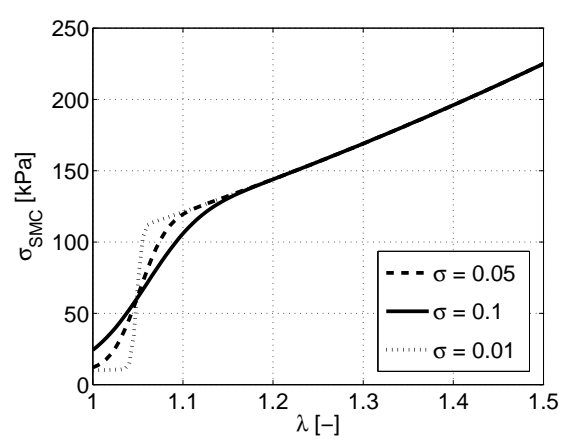

(d) Variation of $\sigma$

Fig. 4 Stress response of smooth muscle cells. Sensitivity analysis for different material parameter values in the strain energy density function. 
Fig. 4 illustrates the stress response of the smooth muscle cell contribution for varying elastic parameters Fig. 4 (a), for varying basal tones Fig. 4(b), for varying critical engagement deformations Fig. 4(c), and for varying half-widths $\sigma 4(\mathrm{~d})$.

\subsection{Collagen behavior}

Various models have been proposed in the literature to characterize the mechanical behavior of collagen fibers. Here, we define the behavior of the individual fibrils by means of the worm-like chain model (Kratky \& Porod, 1949). The WLC describes a continuous flexible rod which presents a conformation state with curvature at ambient temperature and totally rigid at zero Kelvin degrees. This smooth-curved rod presents a preferential direction and has been extensively used to model semiflexible polymers. The worm-like chain model was initially used to model DNA (Bustamante et al., 2003), and was later used for different types of biological tissue. Buehler \& Wong (2007) have used this approach to simulate the behavior of tropocollagen molecules and Kuhl et al. (2005); Garikipati et al. (2006); Alastrue et al. (2010) have adopted it to simulate collagen fibers. Despite the mismatch between the different length scales, all these models have successfully characterized the behavior of the underlying hierarchical structure using the following free energy function of an individual worm-like chain,

$$
\psi\left(\bar{\lambda}_{i}\right)=\left\{\begin{array}{ccc}
\psi_{\mathrm{chn}}+\psi_{\mathrm{rep}} & \text { if } \bar{\lambda}_{i} \geq 1 \\
0 & \text { if } \quad \bar{\lambda}_{i}<1
\end{array}\right.
$$

where

$$
\begin{aligned}
& \psi_{\mathrm{chn}}=\frac{N k \Theta}{4 A} \quad\left[2 \frac{\bar{r}_{i}^{2}}{L}+\frac{L}{1-\bar{r}_{i} / L}-\bar{r}_{i}\right], \\
& \psi_{\text {rep }}=-\ln \left(\bar{\lambda}_{i}^{4 r_{0}^{2}}\right)\left[\frac{1}{L}+\frac{1}{4 r_{0}\left[1-r_{0} / L\right]^{2}}-\frac{1}{4 r_{0}}\right] \quad \text { with } \quad \bar{\lambda}_{i}=\bar{I}_{4}{ }^{1 / 2} \text {, }
\end{aligned}
$$

which is activated only if the fibril is in tension. Here, $N$ represents the chain density or, in other words, the number of molecules per collagen fibril, $k=$ $1.381 \times 10^{-23}(J / K)$ is the Boltzmann constant and $\theta$ is the absolute temperature, which can be set to $\theta=310 K$ for biological tissue. The persistence length $A$ characterizes the ratio between the bending stiffness and the thermal energy, and $L$ is the contour length. Fig. 5 shows a sketch representation of the wormlike chain model. The stretch of each fibril, $\bar{\lambda}_{i}=\left[\mathbf{r}_{i} \cdot \overline{\mathbf{F}}^{t} \cdot \overline{\mathbf{F}} \cdot \mathbf{r}_{i}\right]^{1 / 2}=\bar{I}_{4}^{1 / 2}$, defines the relation between the deformed end-to-end length $\bar{r}_{i}=\bar{\lambda}_{i} r_{0}=\bar{I}_{4}{ }^{1 / 2} r_{0}$ and the initial end-to-end length $r_{0}$. The entropic chain energy $\psi_{\text {chn }}$ denotes the contribution of each individual chain and the repulsive energy $\psi_{\text {rep }}$ is introduced to enforce zero initial stresses in the reference configuration. The latter can be modified to include pre-stresses in the tissue, which have been demonstrated to play an important role in the behavior of blood vessels although we are not including this effect in the present contribution. 


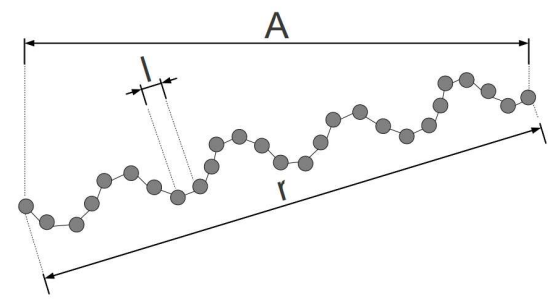

Fig. 5 Representation of a worm-like chain with characteristic end-to-end length $r$, segment length $l$, and persistence length A.

We characterize the collagen behavior at the macroscale, $\Psi_{\text {col }}$, using the microsphere concept and homogenizing the microscale response defined by the collagen fibrils, $\psi\left(\bar{\lambda}_{i}\right)$, by means of a integral over the unit sphere. This homogenization allows us to account for different fibril distributions in space. The microsphere concept has been used successfully to model blood vessels (Caner \& Carol, 2006; Alastrué et al., 2009), elastomers (Miehe et al., 2004) and failure and plasticity (Bazant \& Oh, 1985; Kuhl \& Ramm, 2000). The macroscopic SEDF for collagen is defined as follows.

$$
\Psi_{\mathrm{col}}=\frac{1}{4 \pi} \int_{\mathbb{U}^{2}} \hat{\rho} \psi(\bar{\lambda}) \mathrm{dA} \approx \sum_{i=1}^{i d} \hat{\rho}_{i} w^{i} \psi\left(\bar{\lambda}_{i}\right),
$$

Here, $\mathbb{U}^{2}$ represents the unit sphere, $i d$ is the number of integration directions and $\mathrm{dA}$ is the differential area element of the unit sphere that may be written in terms of the spherical angles $\theta \in[0, \pi)$ and $\phi \in[0,2 \pi)$ as $\mathrm{dA}=\sin (\theta) \mathrm{d} \phi \mathrm{d} \theta$. The normalizing term $A_{\mathbb{U}^{2}}=4 \pi$ is the total area of the unit sphere. The variable $\hat{\rho}$ has been introduced to account for the variability of the collagen fiber distribution, as proposed by (Gasser et al., 2006; Alastrue et al., 2010) in the context of biological tissues. A statistical distribution function characterizes the probability of finding a collagen fibril in a given direction as has been experimentally reported for arterial tissue (Schriefl et al., 2012; Gasser et al., 2012). Here, we adopt a Bingham distribution (Bingham, 1974) to account for the distribution of the individual fibrils, which is given by

$$
\hat{\rho}(\mathbf{r} ; \mathbf{Z}, \mathbf{Q})=\left[F_{000}(\mathbf{Z}, \mathbf{r})\right]^{-1} \operatorname{etr}\left(\mathbf{Z} \cdot \mathbf{Q}^{t} \cdot \mathbf{r} \cdot \mathbf{r}^{t} \cdot \mathbf{Q}\right),
$$

where $\mathbf{r} \in \mathbb{U}^{2}$ are the director vectors, $\operatorname{etr}(\bullet) \equiv \exp (\operatorname{tr}(\bullet))$, and $\mathbf{Z}$ is a diagonal matrix with eigenvalues $\kappa_{1,2,3}$ controlling the concentration distribution. In fact, it is the difference between the pairs $\left[\kappa_{1}-\kappa_{2}\right],\left[\kappa_{1}-\kappa_{3}\right]$ and $\left[\kappa_{2}-\kappa_{3}\right]$ that controls the shape of the function over the unit sphere Waffenschmidt et al. (2011). $\mathbf{Q} \in \mathrm{SO}(3)$ represents the orthogonal local base, which defines the principal directions of the statistical function. Each of the orthogonal vectors $\left(\mathbf{e}_{1}, \mathbf{e}_{2}, \mathbf{e}_{3}\right)$ that defines $\mathbf{Q}$ is directly related to the concentration parameters, $\kappa_{1,2,3}$, respectively. $F_{000}(\mathbf{Z}, \mathbf{r})$ may be written as

$$
F_{000}(\mathbf{Z}, \mathbf{r})=[4 \pi]^{-1} \int_{\mathbb{U}^{2}} \operatorname{etr}(\mathbf{Z}: \mathbf{r} \otimes \mathbf{r}) \mathrm{d} A
$$


In Fig. 6 we show a representation of different Bingham distributions, Fig. 6(a-b), and their corresponding discrete fibrils distributions, Fig.6(c-d). Fig. 7 shows the hierarchical structure from the collagen bundle, left, to the collagen fibril, middle, to the tropocollagen molecule, right, as modeled by the microsphere approach.
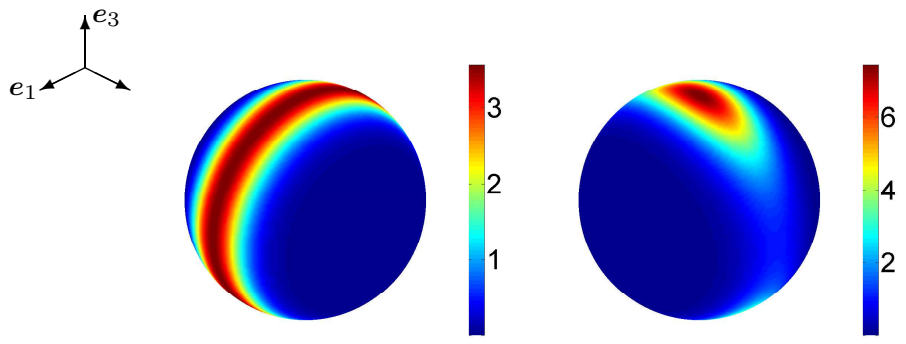

(a) Bingham distribution for (b) Bingham distribution for $\kappa_{1,2,3}=10.0,0.0,10.0$ $\kappa_{1,2,3}=0.0,8.0,10.0$
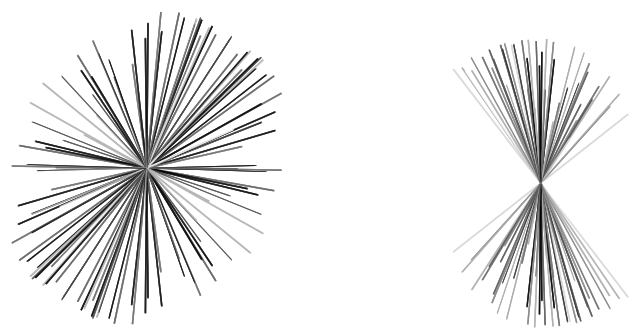

(c) Discrete representation for (d) Discrete representation $\kappa_{1,2,3}=10.0,0.0,10.0 \quad$ for $\kappa_{1,2,3}=0.0,8.0,10.0$

Fig. 6 Bingham and discrete representation of the probabilistic distribution of the collagen bundle.

\subsection{Continuum approach}

The thermodynamic treatment of any type of material whose micro-constituents interact and influence each other, such as biological tissues, is a complex task.

In this section we provide a thermodynamically consistent approach to characterize the material response (Kuhl \& Steinmann, 2003; Menzel, 2004). The material version of the Clasius-Planck inequality for isothermal processes, which is a reasonable assumption for biological tissue with a relatively constant temperature, can be expressed as

$$
\mathcal{D}=-\dot{\Psi}+\frac{1}{2} \mathbf{S}: \dot{\mathbf{C}}-\mathcal{S}_{0} \geq 0
$$

where $\mathcal{D} \geq 0$ is the internal dissipation and $(\dot{\bullet})$ denotes the material time derivative. $\mathbf{S}$ represents the second Piola-Kirchoff stress tensor, and $\mathbf{C}$ is the right Cauchy-Green tensor. 


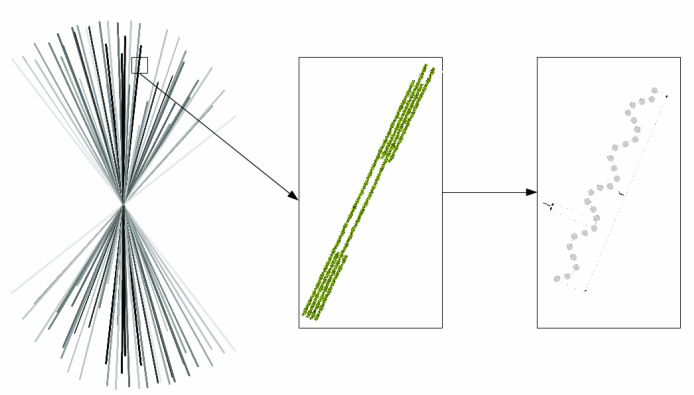

Fig. 7 Hierarchal structure of collagen ranging from the collagen bundle, represented on the left by a 3D space direction, to the collagen fibril in the middle, represented as worm-like chains on the right. The fibril behavior is homogenized and up-scaled by the microsphere-based approach.

To fulfill the second law of thermodynamics, we have added the extra entropy term $\mathcal{S}_{0}$ characteristic for open systems, see, e.g., Truesdell \& Noll (2004) for details. $\Psi=\Psi_{\mathrm{vol}}+\Psi_{\text {iso }}+\Psi_{\text {smc }}+\Psi_{\text {col }}$ is the total SEDF introduced in Eq. (5) as the sum of the volumetric, $\Psi_{\mathrm{vol}}$, isotropic, $\Psi_{\text {iso }}$, smooth muscle cell, $\Psi_{\text {smc }}$ in Eq. (7), and collagen, $\Psi_{\text {col }}$ in Eq.(11) contributions. From its material derivative

$$
\dot{\Psi}=\left[\partial_{\mathbf{C}} \Psi_{\mathrm{vol}}+\partial_{\mathbf{C}} \Psi_{\mathrm{iso}}+\partial_{\mathbf{C}} \Psi_{\mathrm{smc}}+\partial_{\mathbf{C}} \Psi_{\mathrm{col}}\right]: \dot{\mathbf{C}}+\partial_{\rho} \Psi_{\mathrm{col}} \dot{\rho}
$$

we can obtain the expression for the Piola-Kirchhoff stress (see, e.g., Marsden \& Hughes (1994))tensor from Eq. 14 as

$$
\mathbf{S}=2 \partial_{\mathbf{C}} \Psi_{\mathrm{vol}}+2 \partial_{\mathbf{C}} \Psi_{\mathrm{iso}}+2 \partial_{\mathbf{C}} \Psi_{\mathrm{smc}}+2 \partial_{\mathbf{C}} \Psi_{\mathrm{col}}=\mathbf{S}_{\mathrm{vol}}+\mathbf{S}_{\mathrm{iso}}+\mathbf{S}_{\mathrm{smc}}+\mathbf{S}_{\mathrm{col}},
$$

or, more specifically,

$$
\mathbf{S}=2 \frac{\partial \Psi_{\mathrm{vol}}(J)}{\partial J} \frac{\partial J}{\partial \mathbf{C}}+2\left[\frac{\partial \Psi_{\mathrm{iso}}}{\bar{I}_{1}} \frac{\partial \bar{I}_{1}}{\partial \overline{\mathbf{C}}}+\frac{\partial \Psi_{\mathrm{smc}}}{\bar{I}_{4}} \frac{\partial \bar{I}_{4}}{\partial \overline{\mathbf{C}}}+\frac{\partial \Psi_{\mathrm{col}}}{\bar{I}_{4}} \frac{\partial \bar{I}_{4}}{\partial \overline{\mathbf{C}}}\right]: \frac{\partial \overline{\mathbf{C}}}{\partial \mathbf{C}}
$$

Here, it is common to introduce the deviatoric projection tensor $\mathbb{P}=\mathbb{I}-\frac{1}{3} \mathbf{C}^{-1} \otimes$ $\mathbf{C}$, such that $\partial_{\mathbf{C}} \overline{\mathbf{C}}=J^{-2 / 3} \mathbb{P}^{t}$, where $\mathbb{I}=\delta_{i k} \delta_{j l} \mathbf{e}_{i} \otimes \mathbf{e}_{j} \otimes \mathbf{e}_{k} \otimes \mathbf{e}_{l}$ represents the fourth order identity tensor. Accordingly, the purely deviatoric part of the second Piola-Kirchoff stress tensor, $\mathbf{S}_{\mathrm{dev}}$, can be expressed as

$$
\mathbf{S}_{\mathrm{dev}}=J^{-2 / 3} \mathbb{P}: \overline{\mathbf{S}}_{\mathrm{dev}}=J^{-2 / 3} \operatorname{DEV}\left(\overline{\mathbf{S}}_{\mathrm{dev}}\right),
$$

where $\overline{\mathbf{S}}_{\mathrm{dev}}=2 \partial_{\overline{\mathbf{C}}} \Psi_{\mathrm{dev}}$, and $\operatorname{DEV}(\bullet)=\left[\mathbb{I}-\frac{1}{3} \mathbf{C}^{-1} \otimes \mathbf{C}\right]:(\bullet)=\mathbb{P}:(\bullet)$ is the deviatoric operator in the material description.

\section{Methods. Active behavior.}

We now introduce the continuum approach for density growth. In addition to the previous considerations, we now allow the collagen fibril to adapt its 
density, $\rho_{\text {col }}^{i}$, to a given mechanical stimulus, or more specifically, to a given state of energy. To account for growth, the SEDF of each fibril given in Eq. 9 is modified as follows,

$$
\psi_{\mathrm{fib}}\left(\bar{\lambda}_{i}, \rho_{\mathrm{col}}^{i}\right)=\left[\frac{\rho_{\mathrm{col}}^{i}}{\rho_{\mathrm{col}}^{* i}}\right] \psi\left(\bar{\lambda}_{i}\right)
$$

where $\rho_{\text {col }}^{* i}$ is the initial density and $\rho_{\text {col }}^{* i}=\left.\rho_{\text {col }}^{i}\right|_{t=0}$. This results in the following density-weighted collagen bundle energy when integrated over the unit sphere.

$$
\Psi_{\mathrm{col}}=\frac{1}{4 \pi} \int_{\mathbb{U}^{2}} \hat{\rho}\left[\frac{\rho_{\mathrm{col}}}{\rho_{\mathrm{col}}^{*}}\right] \psi(\bar{\lambda}) \mathrm{dA} \approx \sum_{i=1}^{i d} \hat{\rho}_{i} w^{i}\left[\frac{\rho_{\mathrm{col}}^{i}}{\rho_{\mathrm{col}}^{i *}}\right] \psi\left(\bar{\lambda}_{i}\right)
$$

The evaluation of the dissipation inequality in Eq. (14) immediately introduces the density-weighted second Piola-Kirchhoff stress tensor for the collagen bundle,

$$
\mathbf{S}_{\mathrm{col}}=\frac{1}{4 \pi} \int_{\mathbb{U}^{2}} \hat{\rho}\left[\frac{\rho_{\mathrm{col}}}{\rho_{\mathrm{col}}^{*}}\right] \mathbf{S} \mathrm{dA} \approx \sum_{i=1}^{i d} \hat{\rho}_{i} w^{i}\left[\frac{\rho_{\mathrm{col}}^{i}}{\rho_{\mathrm{col}}^{* i}}\right] \mathbf{S}^{i}
$$

where $\mathbf{S}^{i}=J^{-2 / 3} \operatorname{DEV}\left(\overline{\mathbf{S}}^{i}\right)$ with $\overline{\mathbf{S}}^{i}=2 \partial \psi / \partial \overline{\mathbf{C}}$, and thus

$\overline{\mathbf{S}}^{i}=\frac{N K \Theta}{4 A}\left[4 \frac{\bar{\lambda}_{i} r_{0}^{2}}{L}+\frac{r_{0}}{\left[1-\bar{\lambda}_{i} r_{0} / L\right]^{2}}-r_{0}-4 \frac{r_{0}^{2}}{\bar{\lambda}_{i} L}-\frac{r_{0}}{\bar{\lambda}_{i}\left[1-r_{0} / L\right]^{2}}+\frac{r_{0}}{\bar{\lambda}_{i}}\right] \bar{\lambda}_{i}^{-1} \overline{\mathbf{r}}^{i} \otimes \overline{\mathbf{r}}^{i}$

Here, $\overline{\mathbf{r}}_{i}$ denotes the individual director vector of each collagen fibril as introduced in Eqs. (10) and (12). We allow the material density to evolve in time according to the balance of mass for open system thermodynamics, and adopt a source term, $\mathcal{R}$, similar to the one described, in a general form, by Harrigan \& Hamilton (1992); Kuhl \& Steinmann (2003) as

$$
\dot{\rho}=\mathcal{R} \quad \text { with } \quad \mathcal{R}=\left[\frac{\rho}{\rho^{*}}\right]^{-m} \Psi(\mathbf{C})-\Psi^{*},
$$

where the exponent $m$ typically varies between two and three, and $\Psi^{*}$ is the energy of the homeostatic equilibrium state. The overall change in collagen density can be attributed to collagen deposition and collagen degradation in the ECM, in other words, it is caused by the perturbation of the deposition/degradation equilibrium. Collagen turnover is a complex process, in which many interconnected biochemical phenomena affect the final state. Here, we adopt a simplified phenomenological approach to characterize collagen turnover in terms of changes in density. Fig. 8 shows an algorithmic flowchart to analyze the active part of the model, which consists of the passive behavior, the turnover of biological substances, and the remodeling of collagen fibers.

Depending on the basal tone history of the SMCs, which we understand to be the main driving force for these processes, the collagen density will increase 


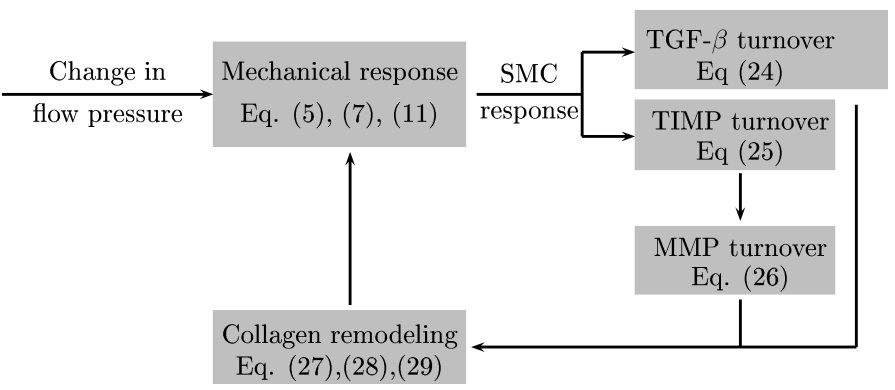

Fig. 8 Algorithmic flowchart to analyze collagen remodeling in response to hypertension.

or decrease. In particular, we hypothesize that an increase in basal tone will increase growth factors such as TGF- $\beta$, responsible for collagen deposition.

$$
\mathcal{R}_{\mathrm{TGF}-\beta}=\dot{\rho}_{\mathrm{TGF}-\beta}=\gamma_{\mathrm{TGF}-\beta}\left[\frac{\rho_{\mathrm{TGF}-\beta}}{\rho_{\mathrm{TGF}-\beta}^{*}}\right]^{-m_{\mathrm{TGF}-\beta}} \Psi_{\mathrm{smc}}\left(\bar{I}_{4}\right)-\Psi_{\mathrm{TGF}-\beta}^{*}
$$

This implies that TGF- $\beta$ will be upregulated, $\mathcal{R}_{\mathrm{TGF}-\beta}>0$, for blood pressures above a characteristic threshold level, $\Psi_{\text {smc }}\left(\bar{I}_{4}\right)>\Psi_{\text {TGF }-\beta}^{*}$, downregulated, $\mathcal{R}_{\mathrm{TGF}-\beta}<0$, for blood pressures below that threshold, $\Psi_{\mathrm{smc}}\left(\bar{I}_{4}\right)<\Psi_{\mathrm{TGF}-\beta}^{*}$, and otherwise remain constant, $\mathcal{R}_{\mathrm{TGF}-\beta}=0$. We further hypothesize that an increase in basal tone will increase tissue inhibitors of metalloproteinase, TIMP, causing a decrease in metalloproteinase, responsible for collagen degradation.

$$
\mathcal{R}_{\mathrm{TIMP}}=\dot{\rho}_{\mathrm{TIMP}}=\gamma_{\mathrm{TIMP}}\left[\frac{\rho_{\mathrm{TIMP}}}{\rho_{\text {TIMP }}^{*}}\right]^{-m_{\mathrm{TIMP}}} \Psi_{\text {smc }}\left(\bar{I}_{4}\right)-\Psi_{\mathrm{TIMP}}^{*}
$$

This implies that TIMP will be upregulated, $\mathcal{R}_{\text {TIMP }}>0$, for blood pressures above a characteristic threshold, $\Psi_{\text {smc }}\left(\bar{I}_{4}\right)>\Psi_{\text {TIMP }}^{*}$, downregulated $\mathcal{R}_{\text {TIMP }}<$ 0 , for blood pressures below the threshold, $\Psi_{\text {smc }}\left(\bar{I}_{4}\right)>\Psi_{\text {TIMP }}^{*}$, and otherwise remain constant, $\mathcal{R}_{\text {TIMP }}=0$. In essence, we consider the changes of TGF- $\beta$ and TIMP as the primary targets, assuming that the up- and downregulation of TGF- $\beta$ and TIMP is driven by the local SMC history. The material parameters $m_{\mathrm{TGF}-\beta}, m_{\mathrm{TIMP}} \in \mathbb{R}^{+}$govern the evolution of TGF- $\beta$ and TIMP respectively, while $\gamma_{\mathrm{TGF}-\beta}, \gamma_{\mathrm{TIMP}} \in \mathbb{R}^{+}$set the sensitivity of these substances to changes in the SMC energy. Our model thus accounts for microstructural changes in terms of the TGF- $\beta$ and TIMP concentrations $\rho_{\text {TGF }-\beta}$ and $\rho_{\text {TIMP }}$. Lastly, we define the source term of the matrix metalloproteinases, which directly change the rate of absorption of collagen. For the sake of simplicity, we hypothesize that it is directly correlated to the source term of TIMP,

$$
\mathcal{R}_{\mathrm{MMP}}=\gamma_{\mathrm{MMP}} \mathcal{R}_{\mathrm{TIMP}}
$$

where $\gamma_{\text {MMP }} \in \mathbb{R}^{-}$defines the sensitivity of MMP to changes in TIMP. With the relevant collagen turnover sources terms defined, we can establish the evolution of the collagen fiber bundles density,

$$
\mathcal{R}_{\mathrm{col}}=\dot{\rho}_{\mathrm{col}}=\gamma_{\mathrm{dep}} \mathcal{R}_{\mathrm{TGF}-\beta}+\gamma_{\mathrm{abs}} \mathcal{R}_{\mathrm{MMP}}
$$


where $\gamma_{\mathrm{dep}}, \gamma_{\mathrm{abs}} \in \mathbb{R}^{+}$denotes the sensitivities of collagen deposition and absorption in response to changes in TGF- $\beta$ and MMP, respectively. In summary, our model can capture three different scenarios, an overall increase, $\mathcal{R}_{\text {col }}>0$, decrease, $\mathcal{R}_{\text {col }}<0$, or maintenance of the collagen content, $\mathcal{R}_{\text {col }}=0$. These scenarios have to be constrained by the following inequalities in order to ensure right collagen turnover.

$$
\mathcal{R}_{\text {col }}\left\{\begin{array}{l}
>0 \text { if } \Psi_{\mathrm{smc}}\left(\bar{I}_{4}\right)>\Psi^{*} \mapsto \gamma_{\mathrm{dep}} / \gamma_{\mathrm{abs}}>\mathcal{R}_{\mathrm{MMP}} / \mathcal{R}_{\mathrm{TGF}-\beta} \\
<0 \text { if } \Psi_{\mathrm{smc}}\left(\bar{I}_{4}\right)<\Psi^{*} \mapsto \gamma_{\mathrm{dep}} / \gamma_{\mathrm{abs}}<\mathcal{R}_{\mathrm{MMP}} / \mathcal{R}_{\mathrm{TGF}-\beta} \\
=0 \text { if } \Psi_{\mathrm{smc}}\left(\bar{I}_{4}\right)=\Psi^{*} \mapsto \gamma_{\mathrm{dep}} / \gamma_{\mathrm{abs}}=\mathcal{R}_{\mathrm{MMP}} / \mathcal{R}_{\mathrm{TGF}-\beta}
\end{array}\right.
$$

To close the set of equations, we evaluate the Clasius-Planck inequality (14)

$$
\mathcal{D}_{\text {int }}=-\partial_{\rho} \Psi_{\text {col }} \dot{\rho}-\mathcal{S}_{0} \geq 0
$$

to identify the extra entropy.

$$
\mathcal{S}_{0} \leq-\mathcal{R}_{\mathrm{col}} \frac{n}{4 \pi} \int_{\mathbb{U}^{2}} \hat{\rho}\left[\frac{1}{\rho_{0}^{*}}\right] \psi \mathrm{dA}
$$

As a summary, in Table 1, we provide the different material parameters presented in this section for the evolution of the collagen density.

Table 1 Collagen turnover model. Relevant material parameters, their physical interpretations, and units.

\begin{tabular}{ccc}
\hline material parameter & physiological interpretation & units \\
\hline$\rho_{\text {TGF }-\beta}^{*}$ & initial density of TGF- $\beta$ & {$[\mu \mathrm{g} / \mathrm{mL}]$} \\
$\rho_{\text {TIMP }}^{*}$ & initial density of TIMP & {$[\mu \mathrm{g} / \mathrm{mL}]$} \\
$\rho_{\text {MMP }}^{*}$ & initial density of MMP & {$[\mu \mathrm{g} / \mathrm{mL}]$} \\
$\rho_{\text {col }}^{*}$ & initial density of collagen & {$[\mu \mathrm{g} / \mathrm{mL}]$} \\
$m_{\text {TGF }-\beta}$ & exponent of TGF- $\beta$ evolution & {$[-]$} \\
$m_{\text {TIMP }}$ & exponent of TIMP evolution & {$[-]$} \\
$\gamma_{\text {TGF }-\beta}$ & sensitivity of TGF- $\beta$ to SMC energy changes & {$[-]$} \\
$\gamma_{\text {TIMP }}$ & sensitivity of TIMP to changes in SMC energy & {$[-]$} \\
$\gamma_{\text {MMP }}$ & sensitivity of MMP to changes in TIMP & {$[-]$} \\
$\gamma_{\text {dep }}$ & sensitivity of collagen deposition to changes in TGF- $\beta$ & {$[-]$} \\
$\gamma_{\text {abs }}$ & sensitivity of collagen absorption to changes in MMP & {$[-]$} \\
\hline
\end{tabular}

\subsection{Computational model}

To solve the non-linear differential equations of collagen deposition and absorption, Eqs. 24 and 25, to characterize the collagen density for the current time step $t$, we adopt a standard Euler backward scheme,

$$
\dot{\rho}_{0}=\left[\rho_{0}^{j+1}-\rho_{0}^{j}\right] / \Delta t
$$

for given initial conditions $\left.\rho_{0}\right|_{t_{0}=0}=\rho_{0}^{*}$. The temporal discretization is given by dividing the time interval $\mathcal{T}$ into $j$ discrete subintervals, $\mathcal{T}=\bigcup_{0}^{j-1}\left[t^{j}, t^{j+1}\right]$, 
with a time increment $\Delta t=t^{j+1}-t^{j} \geq 0$. We transform the evolution equations Eqns. 24 and 25 for TGF- $\beta$ and TIMP into their residual formats.

$$
\begin{aligned}
& \mathrm{R}_{\mathrm{TGF}-\beta}=\rho_{\mathrm{TGF}-\beta}^{j+1}-\rho_{\mathrm{TGF}-\beta}^{j}-\mathcal{R}_{\mathrm{TGF}-\beta} \Delta t \doteq 0 \\
& \mathrm{R}_{\mathrm{TIMP}}=\rho_{\mathrm{TIMP}}^{j+1}-\rho_{\mathrm{TIMP}}^{j}-\mathcal{R}_{\mathrm{TIMP}} \Delta t \doteq 0
\end{aligned}
$$

We solve these equations by applying a local Newton-Raphson iteration, based on a Taylor expansion at $\rho_{\mathrm{TGF}-\beta}^{k}$ and $\rho_{\text {TIMP }}^{k}$ up to terms of first order, see e.g Kuhl et al. (2003); Kuhl \& Steinmann (2003) for more details. To this end, we calculate the derivative of the residuals with respect to the current TGF- $\beta$ and TIMP concentrations.

$$
\begin{gathered}
\left.\frac{\partial \mathrm{R}_{\mathrm{TGF}-\beta}}{\partial \rho_{\mathrm{TGF}-\beta}}\right|_{\rho_{\mathrm{TGF}-\beta}^{k}} ^{-1}=1-\frac{\partial \dot{\rho}_{\mathrm{TGF}-\beta}^{k}}{\partial \rho_{\mathrm{TGF}-\beta}^{k}} \Delta t=1+\frac{m_{\mathrm{TGF}-\beta}}{\rho_{\mathrm{TGF}-\beta}^{k}}\left[\frac{\rho_{\mathrm{TGF}-\beta}^{k}}{\rho_{\mathrm{TGF}-\beta}^{*}}\right]^{-m_{\mathrm{TGF}-\beta}} \Psi_{\mathrm{smc}}\left(\bar{I}_{4}\right) \Delta t \\
\left.\frac{\partial \mathrm{R}_{\mathrm{TIMP}}}{\partial \rho_{\mathrm{TIMP}}}\right|_{\rho_{\mathrm{TIMP}}^{k}} ^{-1}=1-\frac{\partial \dot{\rho}_{\mathrm{TIMP}}^{k}}{\partial \rho_{\mathrm{TIMP}}^{k}} \Delta t=1+\frac{m_{\mathrm{TIMP}}}{\rho_{\mathrm{TIMP}}^{k}}\left[\frac{\rho_{\mathrm{TIMP}}^{k}}{\rho_{\mathrm{TIMP}}^{*}}\right]^{-m_{\mathrm{TIMP}}} \Psi_{\mathrm{smc}}\left(\bar{I}_{4}\right) \Delta t
\end{gathered}
$$

We can then calculate the discrete changes in the TGF- $\beta$ and TIMP concentrations,

$$
\begin{aligned}
& \Delta \rho_{\mathrm{TGF}-\beta}=-\left.\frac{\partial \mathrm{R}_{\mathrm{TGF}-\beta}}{\partial \rho_{\mathrm{TGF}-\beta}}\right|_{\rho_{\mathrm{TGF}-\beta}^{k}} ^{-1} \mathrm{R}_{\mathrm{TGF}-\beta}\left(\rho_{\mathrm{TGF}-\beta}^{k}\right) \\
& \Delta \rho_{\mathrm{TIMP}}=-\left.\frac{\partial \mathrm{R}_{\mathrm{TIMP}}}{\partial \rho_{\mathrm{TIMP}}}\right|_{\rho_{\mathrm{TIMP}}^{k}} ^{-1} \mathrm{R}_{\mathrm{TIMP}}\left(\rho_{\mathrm{TIMP}}^{k}\right)
\end{aligned}
$$

and update the current concentration values.

$$
\begin{aligned}
& \rho_{\mathrm{TGF}-\beta}^{k+1}=\rho_{\mathrm{TGF}-\beta}^{k}+\Delta \rho_{\mathrm{TGF}-\beta} \\
& \rho_{\mathrm{TIMP}}^{k+1}=\rho_{\mathrm{TIMP}}^{k}+\Delta \rho_{\mathrm{TIMP}}
\end{aligned}
$$

Once the local Newton iteration is converged, we can calculate the MMP concentration

$$
\rho_{\mathrm{MMP}}^{j+1}=\gamma_{\mathrm{MMP}} \rho_{\mathrm{TIMP}}^{j+1}=\rho_{\mathrm{MMP}}^{j}+\gamma_{\mathrm{MMP}}\left[\rho_{\mathrm{TIMP}}^{j+1}-\rho_{\mathrm{TIMP}}^{j}\right]
$$

and, finally, the overall collagen content.

$$
\begin{aligned}
& \rho_{\mathrm{col}}^{j+1}=\gamma_{\mathrm{dep}} \rho_{\mathrm{TGF}-\beta}^{j+1}+\gamma_{\mathrm{abs}} \rho_{\mathrm{MMP}}^{j+1}= \\
& =\rho_{\mathrm{col}}^{j}+\gamma_{\mathrm{dep}}\left[\rho_{\mathrm{TGF}-\beta}^{j+1}-\rho_{\mathrm{TGF}-\beta}^{j}\right]+\gamma_{\mathrm{abs}}\left[\rho_{\mathrm{MMP}}^{j+1}-\rho_{\mathrm{MMP}}^{j}\right]
\end{aligned}
$$

Table 2 summarizes the algorithm to compute the update of the local collagen content. 
Table 2 Algorithm to compute the local collagen content using an implicit Euler backward scheme.

\section{Input: $\mathbf{F}^{j+1}$, internal variables at time $j$}

I Evaluate kinematics $\mathbf{C}^{j+1}$ and constitutive equations $\Psi^{j+1}, \mathbf{S}^{j+1}$

II Check state of SMC

IF $\left(\Psi_{\mathrm{smc}}^{j+1} \neq \Psi^{*}\right)$ THEN GO TO III

ELSE GO TO IV

III Local Newton iteration. WHILE $\mathrm{R}_{\rho_{\mathrm{TGF}-\beta}}>$ tol or $\mathrm{R}_{\rho_{\mathrm{TIMP}}}>$ tol

Calculate local TGF- $\beta$ and TIMP residuals

$$
\begin{aligned}
& \mathrm{R}_{\mathrm{TGF}-\beta}=-\rho_{\mathrm{TGF}-\beta}^{j+1}+\rho_{\mathrm{TGF}-\beta}^{j}+\mathcal{R}_{\mathrm{TGF}-\beta} \Delta t \doteq 0 \\
& \mathrm{R}_{\mathrm{TIMP}}=-\rho_{\mathrm{TIMP}}^{j+1}+\rho_{\mathrm{TIMP}}^{j}+\mathcal{R}_{\mathrm{TIMP}} \quad \Delta t \doteq 0
\end{aligned}
$$

Calculate local TGF- $\beta$ and TIMP tangents

$$
\begin{aligned}
& \mathrm{K}_{\mathrm{TGF}-\beta}=1+\frac{m_{\mathrm{TGF}-\beta}}{\rho_{\mathrm{TGF}-\beta}^{k}}\left[\frac{\rho_{\mathrm{TGF}-\beta}^{k}}{\rho_{\mathrm{TGF}-\beta}^{*}}\right]^{-m_{\mathrm{TGF}-\beta}} \Psi_{\mathrm{smc}}\left(\overline{I_{4}}\right) \Delta t \\
& \mathrm{~K}_{\mathrm{TIMP}}=1+\frac{m_{\mathrm{TIMP}}}{\rho_{\mathrm{TIMP}}^{k}}\left[\frac{\rho_{\text {TIMP }}^{k}}{\rho_{\text {TIMP }}^{*}}\right]^{-m_{\mathrm{TIMP}}} \Psi_{\mathrm{smc}}\left(\bar{I}_{4}\right) \Delta t
\end{aligned}
$$

Update TGF- $\beta$ and TIMP concentrations

$$
\begin{aligned}
& \rho_{\mathrm{TGF}-\beta}^{\mathrm{j}+1} \leftarrow \rho_{\mathrm{TGF}-\beta}^{\mathrm{j}+1}-\mathrm{R}_{\rho_{\mathrm{TGF}-\beta}} / \mathrm{K}_{\rho_{\mathrm{TGF}-\beta}} \\
& \rho_{\mathrm{TIMP}}^{\mathrm{j}+1} \leftarrow \rho_{\mathrm{TIMP}}^{\mathrm{j}+1}-\mathrm{R}_{\rho_{\mathrm{TIMP}}} / \mathrm{K}_{\rho_{\mathrm{TIMP}}}
\end{aligned}
$$

IV Update MMP concentration and collagen content

$$
\begin{aligned}
& \rho_{\mathrm{MMP}}^{j+1}=\gamma_{\mathrm{MMP}} \rho_{\mathrm{TIMP}}^{j+1} \\
& \rho_{\mathrm{col}}^{j+1}=\gamma_{\mathrm{dep}} \rho_{\mathrm{TGF}-\beta}^{j+1}+\gamma_{\mathrm{abs}} \rho_{\mathrm{MMP}}^{j+1}
\end{aligned}
$$

$\mathrm{V}$ Calculate stresses $\mathbf{S}^{t+1}$

Output: $\mathbf{S}^{t+1}, \rho_{\mathrm{TGF}-\beta}^{t+1}, \rho_{\mathrm{TIMP}}^{t+1} \rho_{\mathrm{MMP}}^{t+1}$ and $\rho_{\mathrm{Col}}^{t+1}$

\section{Results}

To illustrate the features of our model we present numerical examples of selected test cases. We set the material parameters of the passive behavior of the tissue for the wormlike chain model (Garikipati et al., 2006; Kuhl et al., 2005; Alastrue et al., 2010) to $L=1.532, r_{0}=1.072, k=1.381 \times 10^{-23}(\mathrm{~J} / \mathrm{K})$, $N=1.38 \times 10^{21}, \theta=310 K$ and $A=1.107$, as adapted from Alastrue et al. (2010). The concentration parameters for the Bingham distribution are set to $\kappa_{1,2,3}=0.0,56.81,58.70$. For illustrative purposes, we set the SMC parameters to $\mu_{\mathrm{smc}}=100, S_{\mathrm{bsl}}=0.1, \mu=1.1$ and $\sigma=0.01$.

\subsection{Model problem of density evolution}

In Fig. 9 and Fig. 10, we present a sensitivity analysis of the material parameters involved in the evolution of the TGF $-\beta$ content for baseline values of $\mathrm{m}=2, \rho_{\mathrm{TGF}-\beta}^{*}=1.0, \gamma_{\mathrm{TGF}-\beta}=1.0$ and $\Psi_{\mathrm{TGF}-\beta}^{*}=1.5$. We study the problem 


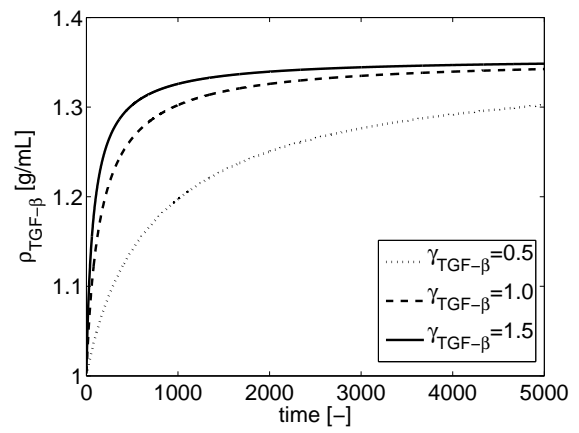

(a) Variation of $\gamma_{\mathrm{TGF}-\beta}$

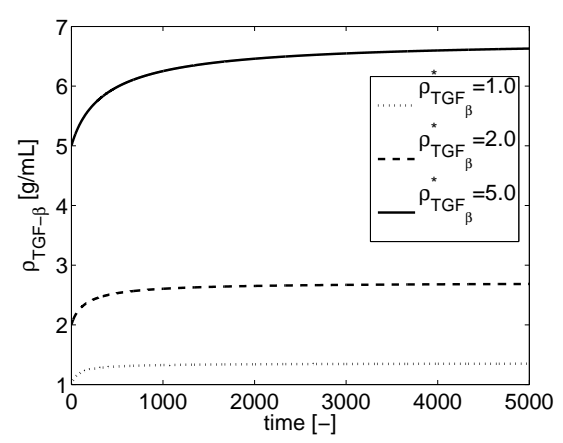

(c) Variation of $\rho_{\mathrm{TGF}-\beta}^{*}$

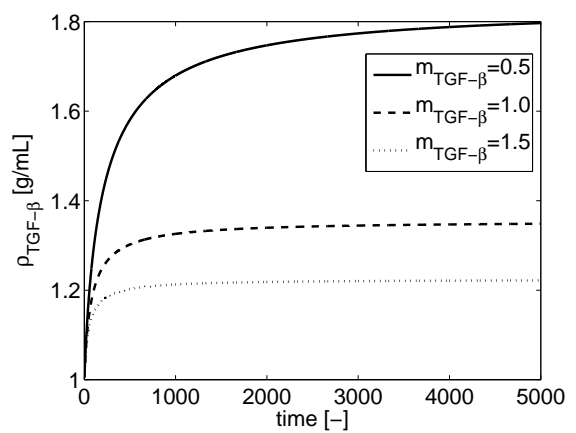

(b) Variation of $m_{\mathrm{TGF}-\beta}$

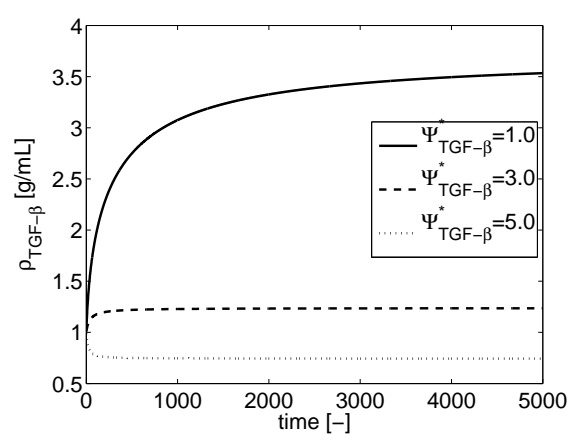

(d) Variation of $\Psi_{\mathrm{TGF}-\beta}^{*}$

Fig. 9 Sensitivity of TGF $-\beta$ content $\rho_{\mathrm{TGF}-\beta}$ with respect to sensitivity parameter $\gamma_{\mathrm{TGF}-\beta}$, evolution exponent $m_{\mathrm{TGF}-\beta}$, initial value $\rho_{\mathrm{TGF}-\beta}^{*}$, and saturation value $\Psi_{\mathrm{TGF}-\beta}^{*}$.

for $j=5000$ discrete time steps of $\Delta t=1$. We illustrate the sensitivity of the TGF $-\beta$ concentration for varying sensitivity parameters $\gamma_{\text {TGF- } \beta}$ in Fig. 9(a), for varying exponents $m_{\text {TGF- } \beta}$ in Fig.9(b), for varying initial values $\rho_{\text {TGF- } \beta}^{*}$ in Fig $9(\mathrm{c})$, and for varying saturation values $\Psi_{\mathrm{TGF}-\beta}^{*}$ in Fig $9(\mathrm{~d})$. In Fig. 10, we illustrate the sensitivity of the TGF $-\beta$ concentration for varying elastic moduli of the SMC $\mu_{\text {smc }}$ in Fig. 10(a), for varying basal tone $S_{\mathrm{bsl}}$ in Fig. 10(b), and for varying active response of SMC $\mu$ and $\sigma$ in Figs. 10(c) and 9(d). Next, we further illustrate the case presented in 10 (c) with $\mu=1.1$. Fig. 11 shows the changes in collagen content in the case of plain collagen deposition without absorption. This implies that the collagen density is affected by changes in TGF- $\beta$, while TIMP and MMP remain constant. In Fig. 11(a) we show the deposition of collagen over time. Figs. 12(b), (c), and (d) illustrate the corresponding evolution of the Bingham distribution at three discrete time points, $t=0,500$, and 5000. We observe an increase in collagen density close to $75 \%$, which is confirmed by the evolution of the Bingham distribution. Fig. 12 shows the changes in collagen content in the case of plain collagen absorption without deposition. This implies that the collagen density is affected by changes in TIMP and MMP, while TGF- $\beta$ remains constant. In Fig. 12(a) displays the 


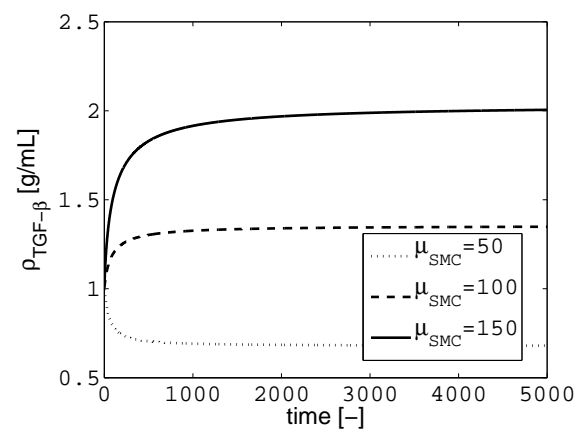

(a) Variation of $\mu_{\mathrm{SMC}}$

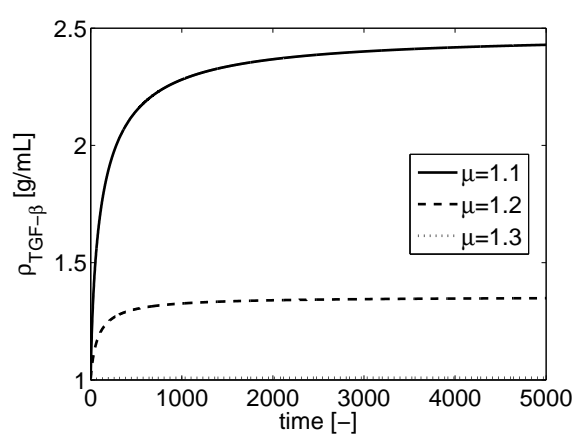

(c) Variation of $\mu$

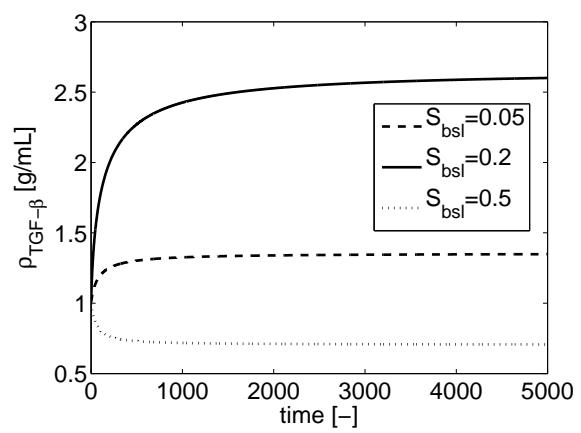

(b) Variation of $S_{b s l}$

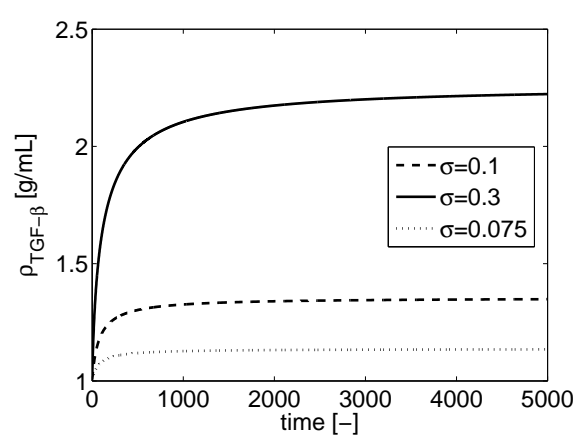

(d) Variation of $\sigma$

Fig. 10 Sensitivity of TGF $-\beta$ content $\rho_{\text {col }}$ with respect to elastic modulus of the SMC $\mu_{\mathrm{smc}}$, basal tone $S_{\mathrm{bsl}}$, and active response of SMC $\mu$ and $\sigma$.

absorption of collagen over time. Figs. 12(b), (c), and (d) illustrate the corresponding evolution of the Bingham distribution at three discrete time points, $t=0,500$, and 5000. In Fig. 12 shows a decreases in collagen density of almost 90\%. The Bingham distribution confirms this collagen decrease over time. In Fig. 13 we summarize the overall evolution of the collagen content affected by TGF- $\beta$, TIMP and MMP. For the chosen set of parameters, the amount of collagen decreases over time. This implies that the rate of collagen absorption is greater than the rate of collagen deposition. Fig 14 displays a graphic sketch of the collagen turnover. At low pressure levels, collagen absorption is greater than collagen deposition, and the overall amount of collagen decreases. As a result, the extracellular matrix weakens over time. Finally, to demonstrate how the mechanical properties are affected by changes in TGF- $\beta$ and TIMP concentration, we illustrate the resulting stress for the case of uniaxial tension, stretching in $\mathrm{e}_{3}$, in Fig. 15. The solid line illustrates the stress response in the case of collagen deposition initiated through an increase in TGF- $\beta$. The dashdotted line illustrates the stress response in the case of collagen absorption initiated through an increase in TIMP. The dotted line situated in between illustrates the combined case of collagen deposition and absorption. The material is stiffest in the deposition case and weakest in the absorption case. The 


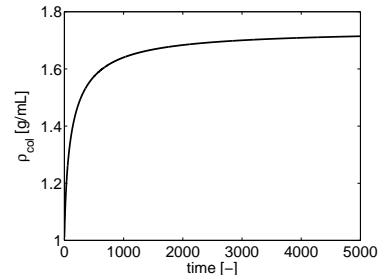

(a) Evolution of the density

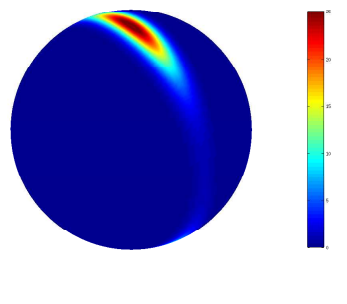

(b) Statistical distribution at $t=0$

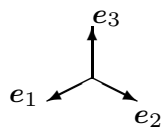
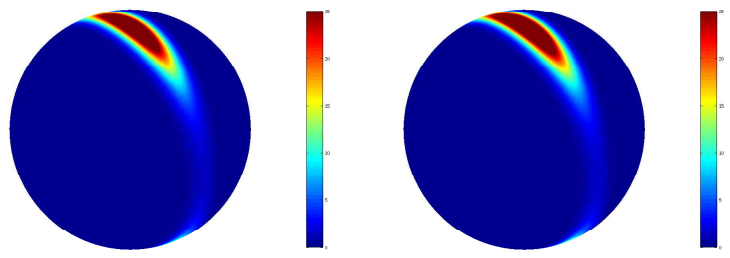

(c) Statistical distribution at (d) Statistical distribution at $t=500$ $t=5000$

Fig. 11 Evolution of the collagen content in the case of plain collagen deposition without absorption. Collagen is affected exclusively by alterations in the TGF- $\beta$ content, while TIMP and MMP remain constant.

combined case lies in between but slightly below the baseline case as indicated through the dashed line. This confirms an overall decrease in collagen concentration associated with a softer material response.

\subsection{Hypertension-induced collagen deposition and absorption}

As discussed in the introductory section, the turnover of collagen in disease conditions can be related to changes in the rates of deposition and absorption. However, the net result of collagen production and removal strongly depends on the type of tissue and on the level of hypertension. In this section we test our model against real experimental results (see Laviades et al. (1998)), who investigated alterations in collagen type I, matrix metalloproteinase, and inhibitors of matrix metalloproteinase. The experimental study found an increase of $178.9 \%$, from $641 \pm 31 \mathrm{ng} / \mathrm{mL}$ to $1147 \pm 55$, in total TIMP-1 and a decrease in total MMP-1 density of $18.07 \%$, from $56 \pm 2 \mathrm{ng} / \mathrm{mL}$ to $47 \pm 1 \mathrm{ng} / \mathrm{mL}$. The authors point out that both TGF- $\beta$ and collagen content are upregulated in hypertensive patients. Given the normal baseline concentration of TGF- $\beta$ of $35 \mathrm{ng} / \mathrm{mL}$ (Schaan et al., 2007), and its 4.2-fold increase in hypertensive patients Porreca et al. (1997), we can compute the increase in collagen to the upregulation of TGF- $\beta$. In addition, we take into account the results given in Diez et al. (1995), where the serum concentration of procollagen peptides was 


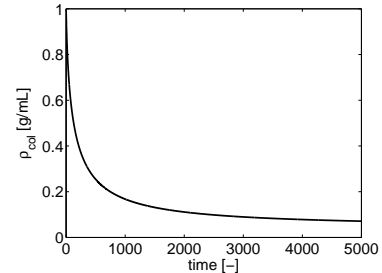

(a) Evolution of the density

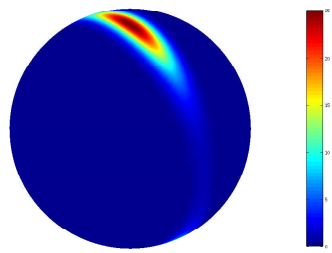

(b) Statistical distribution at $t=0$<smiles>[CH]C([CH2])[GeH3]</smiles>
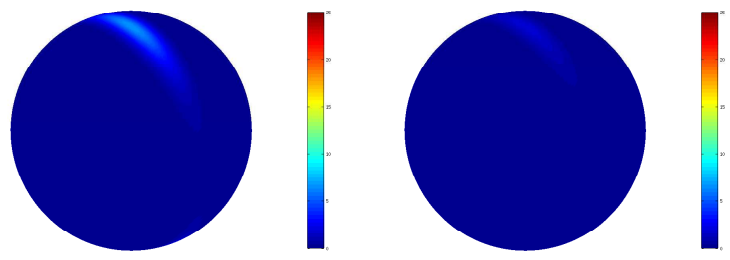

(c) Statistical distribution at (d) Statistical distribution at $t=500$ $t=5000$

Fig. 12 Evolution of the collagen content in the case of plain collagen absorption without deposition. Collagen is affected exclusively by alterations in the TIMP and MMP content, while TGF- $\beta$ remains constant.

examined. The authors studied the concentration of procollagen type I carboxy terminal peptide (PIP), which has been proposed as a marker of collagen type I synthesis. It displayed an increase of $28 \%$. The same authors (Laviades et al., 1998) found that the carboxy-terminal telopeptide of collagen type I (CITP), a marker of extracellular collagen degradation, increased by approximately a $9 \%$.

Accordingly, we simulate the case of hypertension, to explore to what extent our model is capable of reproducing these experimental findings. To quantify the collagen turnover in response to an increase in TGF- $\beta$ and a decrease in MMP, we compute the deposition and absorption rates $\dot{\rho}_{\text {col }}^{+}=\gamma_{\text {abs }} \dot{\rho}_{\text {TGF }-\beta}$ and $\dot{\rho}_{\text {col }}^{-}=\gamma_{\text {dep }} \dot{\rho}_{\text {MMP }}$. The material parameters are summarized in Table 3.

In Fig. 16, we summarize the evolution of the TGF- $\beta$ (a), TIMP (b) and MMP (c) contents in response to hypertension. In Fig. 17 we illustrate the impact of these changes on the evolution of the overall collagen content. With the help of the studies reported in the literature, we can correlate the collagen synthesis to the TGF- $\beta$ concentration as shown in Fig. 17(a). Fig. 17(b) illustrates collagen degradation due to the decrease in MMP. Fig. 17(c) displays the overall turnover of collagen, confirming the increase in collagen content characteristic in hypertensive patients. In Figs. 17(d) and (e), we show the resulting Bingham distribution for a healthy and hypertensive artery wall, i.e., 


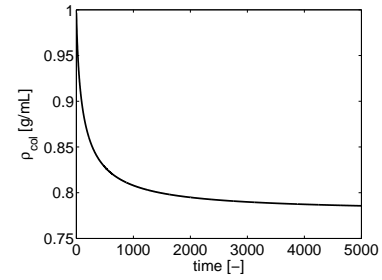

(a) Evolution of the density

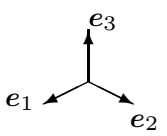
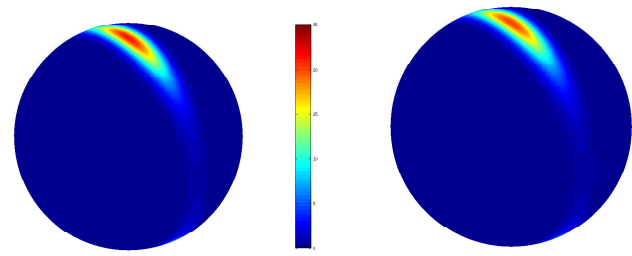

(c) Statistical distribution at $t=500$

(d) Statistical distribution at $t=5000$

Fig. 13 Evolution of the collagen content in the combined case of collagen absorption and deposition. Collagen is affected by alterations in both TGF- $\beta$ and TIMP.

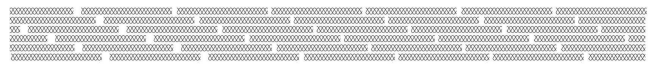

(a) Fibril structure at $t=0$

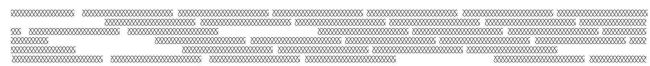

(b) Fibril structure at $t=500$

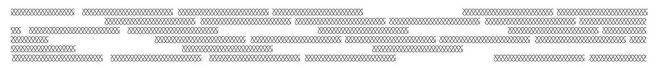

(c) Fibril structure at $t=5000$

Fig. 14 Structural evolution of collagen fibrils in response to progressive collagen turnover. The illustrated case demonstrates the overall absorption of collagen in the case of low blood pressure due to the disassembly of tropomolecules within the collagen fibril . It is characterized through a low collagen deposition and a high collagen absorption resulting in a net decrease in collagen density.

in the initial baseline state at $t=0$ and in the final state of the simulation at $t=5000$. 


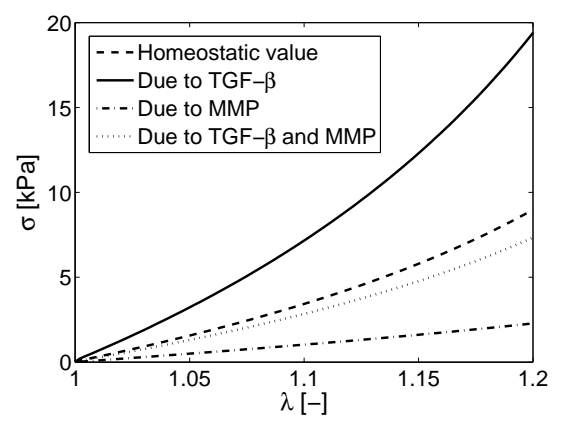

Fig. 15 Evolution of stress in the case of collagen deposition initiated through an increase in TGF- $\beta$, solid line, collagen absorption initiated through an increase in TIMP and MMP, dash-dotted line, and combined case of deposition and absorption initiated through an increase in TGF- $\beta$, TIMP, and MMP, dotted line situated between both cases. For comparison, the baseline stress value is illustrated through the dashed line.

Table 3 Collagen turnover model. Material parameters for hypertensive case study.

\begin{tabular}{ccc}
\hline material parameter & value & units \\
\hline$\rho_{\text {TGF }-\beta}^{*}$ & 0.035 & {$[\mu \mathrm{g} / \mathrm{mL}]$} \\
$\rho_{\text {TIMP }}^{*}$ & 0.0459 & {$[\mu \mathrm{g} / \mathrm{mL}]$} \\
$\rho_{\mathrm{MMP}}^{*}$ & 0.0353 & {$[\mu \mathrm{g} / \mathrm{mL}]$} \\
$\rho_{\text {col }}^{*}$ & 1 & {$[\mathrm{~g} / \mathrm{mL}]$} \\
$m_{\text {TGF }-\beta}$ & 0.04 & {$[-]$} \\
$m_{\text {TIMP }}$ & 0.086 & {$[-]$} \\
$\gamma_{\text {TGF }-\beta}$ & 0.2 & {$[-]$} \\
$\gamma_{\text {TIMP }}$ & 0.4 & {$[-]$} \\
$\gamma_{\text {dep }}$ & -0.0075 & {$[-]$} \\
$\gamma_{\text {abs }}$ & 90000 & {$[-]$} \\
\hline
\end{tabular}

\section{Conclusions}

We have presented a theoretical and computational model for collagen turnover in soft biological tissue in response to changes in its mechanical environment. We have focused on cardiovascular tissues such as blood vessels, and have hypothesized that changes in collagen density can be attributed primarily to two pathways of mechanotransduction. Collagen deposition was correlated to the concentration of the growth factor TGF- $\beta$, with increasing concentrations increasing the collagen deposition rate. Collagen absorption was correlated to tissue inhibitors of metalloproteinase TIMP, with increasing TIMP concentrations decreasing metallopreteinase, thereby increasing the collagen absorption rate. These processes are controlled mainly by smooth muscle and endothelial cell activity.

In this paper, we have established a mathematical model for density growth of individual collagen bundles. We have introduced a computational algorithm to solve an underlying set of non-linear equations for the TGF- $\beta$ and TIMP densities, from which we can then extract the overall collagen turnover rate. To characterize the passive behavior of the vessel wall, we have used a hierarchical multiscale model to characterize the constitutive response at the different scales. At the microscopic scale, we have used a worm-like chain model to 


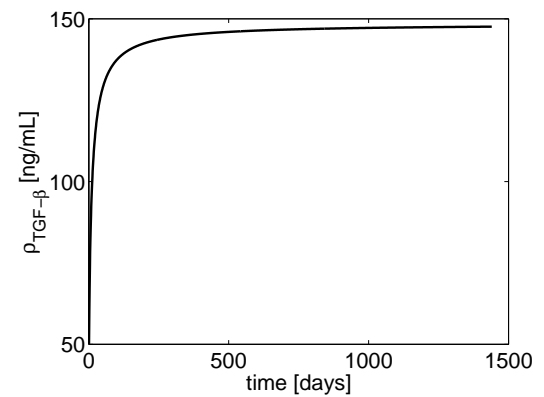

(a) Evolution of TGF- $\beta$

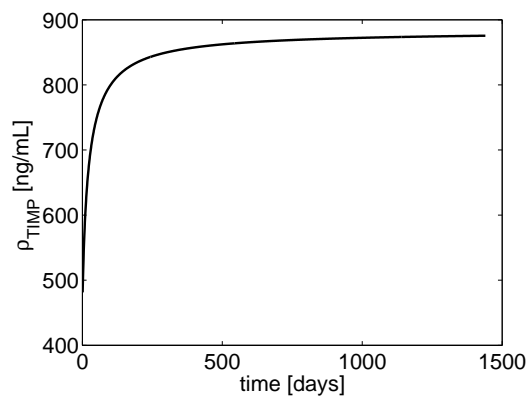

(b) Evolution of TIMP

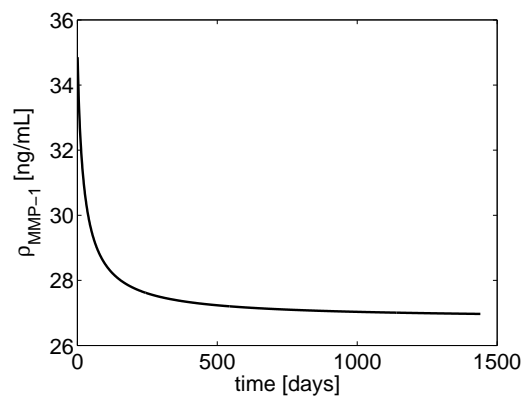

(c) Evolution of MMP

Fig. 16 Evolution of TGF- $\beta$, TIMP, and MMP concentrations involved in progressive collagen turnover.

simulate the behavior of individual collagen fibrils, made up of tropocollagen molecules. While conceptually different, both collagen fibrils and tropocollagen molecules can be modeled with the wormlike chain model with good results in both cases, although with different material parameters. This approximation can be accepted upon the assumption that tropocollagen molecules assemble into fibrils with very high bonds, which give them a behavior similar to the tropocollagen molecule itself, although with a different stiffness. At the mesoscopic scale, the collagen bundle is composed of aggregates of collagen fibrils. In order to scale up from fibrils to collagen bundles, we adopted a microspherebased approach which has been applied successfully in this context in the past. In addition, we have enhanced the classical microsphere concept with a statistical distribution function to account for the dispersion of the fibrils. At the macroscopic scale, we have formulated an enhanced continuum approach which allows for changes in the local collagen content, adopting concepts from macroscopic growth models for vessels and bones. Our model is capable of representing the main constituents of blood vessels, i.e., water, elastin, smooth muscle cells, and collagen. Smooth muscle cells have been characterized by a strain energy density function which takes into account the active behavior of the cells. This strain energy density function of the smooth muscle cells has been used as the driving force for collagen turnover. Based on this stimulus, we have solved for the concentration changes in TGF- $\beta$ and TIMP responsible 

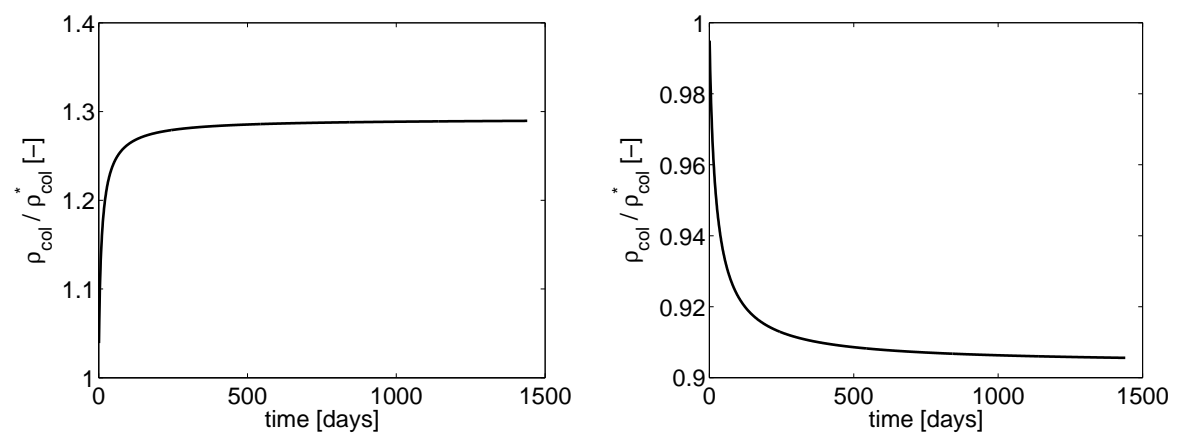

(a) Evolution of collagen density in response (b) Evolution of collagen density in response to increase in TGF- $\beta$ to decrease of MMP
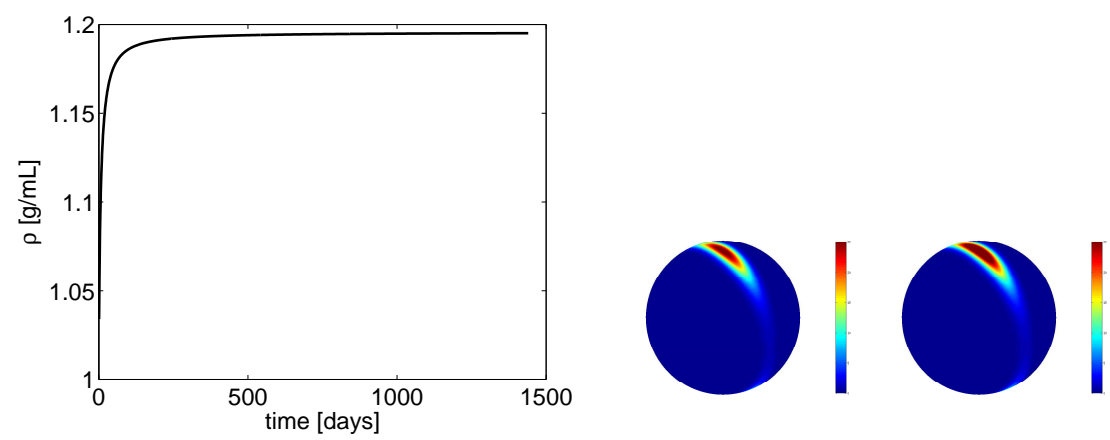

(c) Evolution of the collagen density in re- (d) Initital Bing- (e) Final Bingham sponse to both increase in TGF- $\beta$ and decrease ham distribution at distribution at in MMP

$\mathrm{t}=0 \quad \mathrm{t}=5000$

Fig. 17 Evolution of the collagen content in hypertensive patients driven by an increase in TGF- $\beta$ and a decrease in MMP.

for the turnover of MMP and, ultimately, for the deposition and absorption of collagen. In this manner, this contribution provides microstructural insight into the mechanotransduction pathways related to collagen turnover. We have illustrated these pathways by means of a simple model problem with generic material parameter values, and by means of a clinically motivated test case of hypertension. A good qualitative and quantitative agreement with the experimental findings was achieved.

Despite the intuitive agreement with common clinical findings, this model is only a first prototype that is not free of limitations. First and foremost, the current material parameters have not been calibrated and validated correctly. Rather, they have been introduced generically to explore first trends. The main issue is, of course, the lack of a complete experimental database to calibrate the model parameters correctly and validate the model. Unfortunately, most existing studies correlate the evolution of collagen only to one single event and neglect the interaction of the different mechanotransduction pathways involved in the overall process. The parameter calibration is further complicated by the huge variability between different species, between different individuals 
within the same species, and even between different locations within the same individual. Our main goal in the future is to perform well-defined experiments, at least in one species, at a predefined location.

Another important aspect is the complex interplay of the individual mechanotransduction pathways which ultimately converge in alterations in collagen turnover rates. Collagen turnover in response to hypertension is a highly complex process in which many substances are involved. First, the proposed smooth muscle cell approach could be improved by including models to account for discrete changes in calcium and potassium concentrations, ideally models that correlate these changes to ion channel dynamics. This is, of course, a complex task, and out of the scope of the present study. In terms of the substances that are directly involved in the collagen turnover, growth factors and matrix metalloproteinases, a better understanding is needed of the qualitative and quantitative correlation between smooth muscle cell activity and up- and downregulation of these events. In addition, it is known that tropocollagen molecules are absorbed by breaking into $1 / 4$ and $3 / 4$ pieces of the molecule before fully dissolving into the tissue. A more detailed model of how these rupture processes occur would further improve our collagen turnover model. From a computational point of view, a logical next step would be to include the present model in a nonlinear finite element setting which will allow the simulation of arbitrary geometries and heterogeneous parameter distributions of real patient-specific vessels.

\section{References}

D. J. Adams, et al. (1989). 'Ion Channels and Regulation of Intracellular Calcium In Vascular Endothelial-cells'. Faseb Journal 3(12):2389-2400.

V. Alastrue, et al. (2010). 'On the use of the Bingham statistical distribution in microsphere-based constitutive models for arterial tissue'. Mech Res Commun 37(8):700-706.

V. Alastrué, et al. (2009). 'Anisotropic micro-sphere-based finite elasticity applied to blood vessel modelling'. J Mech Phys Solids 57(1):178-203.

D. Ambrosi, et al. (2011). 'Perspectives on biological growth and remodeling'. $J$ Mech Phys Solids 59(4):863-883.

G. A. Ateshian (2007). 'On the theory of reactive mixtures for modeling biological growth'. Biomech Model Mechan 6(6):423-445.

D. R. Baselt, et al. (1993). 'Subfibrillar Structure of Type-i Collagen Observed By Atomic-force Microscopy'. Biophys J 65(6):2644-2655.

Z. P. Bazant \& B. H. Oh (1985). 'Microplane Model For Progressive Fracture of Concrete and Rock'. J Eng Mech-ASCE 111(4):559-582.

J. Bella, et al. (1994). 'Crystal-structure and Molecular-structure of A Collagen-like Peptide At 1.9-angstrom Resolution'. Science 266(5182):7581.

J. A. Bevan \& I. Laher (1991). 'Pressure and Flow-dependent Vascular Tone'. Faseb Journal 5(9):2267-2273.

A. Bhattacharjee \& M. Bansal (2005). 'Collagen structure: The Madras triple helix and the current scenario'. IUBMB Life 57(3):161-172. 
C. Bingham (1974). 'An Antipodally Symmetric Distribution on the Sphere'. Ann Stat 2(6):1201-1225.

J. E. Bishop (1998). 'Regulation of cardiovascular collagen deposition by mechanical forces'. Mol Med Today 4(2):69-75.

J. E. Bishop \& G. Lindahl (1999). 'Regulation of cardiovascular collagen synthesis by mechanical load'. Cardiovasc Res 42(1):27-44.

W. Bode, et al. (1999). 'Structural properties of matrix metalloproteinases'. Cell Mol Life Sci 55(4):639-652.

W. A. Border \& N. A. Noble (1994). 'Transforming Growth-factor-beta In Tissue Fibrosis'. New Engl J Med 331(19):1286-1292.

C. J. Boyle, et al. (2011). 'In Silico Prediction of the Mechanobiological Response of Arterial Tissue: Application to Angioplasty and Stenting'. Journal of Biomechanical Engineering-transactions of the Asme 133(8):081001.

J. E. Brayden \& M. T. Nelson (1992). 'Regulation of Arterial Tone By Activation of Calcium-dependent Potassium Channels'. Science 256(5056):532535.

M. J. Buehler \& S. Y. Wong (2007). 'Entropic elasticity controls nanomechanics of single tropocollagen molecules RID C-4580-2008'. Biophys J 93(1):37-43.

C. Bustamante, et al. (2003). 'Ten years of tension: single-molecule DNA mechanics'. Nature 421(6921):423-427.

R. P. Butt, et al. (1995). 'Collagen Production and Replication By Cardiac Fibroblasts Is Enhanced In Response To Diverse Classes of Growth-factors'. Eur J Cell Biol 68(3):330-335.

F. C. Caner \& I. Carol (2006). 'Microplane Constitutive Model and Computational Framework for Blood Vessel Tissue'. J Biomech Eng 128(3):419-427.

O. A. Carretero \& S. Oparil (2000). 'Essential hypertension Part I: Definition and etiology'. Circulation 101(3):329-335.

L. D. Chung, et al. (2004). 'Collagenase unwinds triple-helical collagen prior to peptide bond hydrolysis'. Embo Journal 23(15):3020-3030.

B. D. Cumming, et al. (2010). 'A mathematical model of wound healing and subsequent scarring'. Journal of the Royal Society Interface 7(42):19-34.

M. J. Davis \& M. A. Hill (1999). 'Signaling mechanisms underlying the vascular myogenic response'. Physiol Rev 79(2):387-423.

J. Diez, et al. (1995). 'Increased Serum Concentrations of Procollagen Peptides In Essential-hypertension - Relation To Cardiac Alterations'. Circulation 91(5):1450-1456.

N. Famaey \& J. V. Sloten (2008). 'Soft tissue modelling for applications in virtual surgery and surgical robotics'. Computer Methods In Biomechanics and Biomedical Engineering 11(4):351-366.

N. Famaey, et al. (2011). 'A three-constituent damage model for arterial clamping in computer-assisted surgery' Submitted for publication.

G. B. Fields (1991). 'A Model For Interstitial Collagen Catabolism By Mammalian Collagenases'. J Theor Biol 153(4):585-602.

P. J. Flory (1961). 'Thermodynamic relations for high elastic materials'. T Faraday Soc 57:829-838.

B. Folkow (1949). 'Intravascular Pressure As A Factor Regulating the Tone of the Small Vessels'. Acta Physiol Scand 17(4):289-310. 
B. Folkow (1982). 'Physiological-aspects of Primary Hypertension'. Physiol Rev $62(2): 347-504$.

B. Folkow, et al. (1958). 'Adaptive Structural Changes of the Vascular Walls In Hypertension and Their Relation To the Control of the Peripheral Resistance'. Acta Physiol Scand 44(3-4):255-272.

P. Fratzl (2008). Collagen: Structure and Mechanics. Springer (New York).

P. Fratzl, et al. (2004). 'Structure and mechanical quality of the collagenmineral nano-composite in bone'. J Mater Chem 14(14):2115-2123.

E. D. Freis (1960). 'Hemodynamics of Hypertension'. Physiol Rev 40(1):27-54.

Y. Fung \& S. Liu (1989). 'Change of residual strains in arteries due to hypertrophy caused by aortic constriction'. Circ Res 65(5):1340-1349.

Z. S. Galis \& J. J. Khatri (2002). 'Matrix metalloproteinases in vascular remodeling and atherogenesis - The good, the bad, and the ugly'. Circ Res 90(3):251-262.

Z. S. Galis, et al. (1994). 'Cytokine-stimulated Human Vascular Smooth-muscle Cells Synthesize A Complement of Enzymes Required For Extracellularmatrix Digestion'. Circ Res 75(1):181-189.

K. Garikipati, et al. (2006). 'Biological remodelling: Stationary energy, configurational change, internal variables and dissipation'. J Mech Phys Solids 54(7):1493-1515.

T. C. Gasser, et al. (2012). 'Spatial orientation of collagen fibers in the Abdominal Aortic Aneurysm's wall and its relation to wall mechanics.'. Acta Biomater Accepted for publication.

T. C. Gasser, et al. (2006). 'Hyperelastic modelling of arterial layers with distributed collagen fibre orientations'. J Roy Soc Interface 3:15-35.

A. Gautieri, et al. (2011). 'Hierarchical Structure and Nanomechanics of Collagen Microfibrils from the Atomistic Scale Up'. Nano Letters 11(2):757-766.

R. Gleason \& J. Humphrey (2005). 'Effects of a sustained extension on arterial growth and remodeling: a theoretical study'. J Biomech 38(6):1255-1261.

R. L. Gleason \& J. D. Humphrey (2004). 'A mixture model of arterial growth and remodeling in hypertension: Altered muscle tone and tissue turnover'. $J$ Vasc Res 41(4):352-363.

J. H. Haga, et al. (2007). 'Molecular basis of the effects of mechanical stretch on vascular smooth muscle cells'. J Biomech 40(5):947-960.

P. Hamet, et al. (1991). 'Transforming Growth-factor Beta-1 Expression and Effect In Aortic Smooth-muscle Cells From Spontaneously Hypertensive Rats'. Hypertension 17(6):896-901.

T. P. Harrigan \& J. J. Hamilton (1992). 'An Analytical and Numerical Study of the Stability of Bone Remodeling Theories - Dependence On Microstructural Stimulus'. J Biomech 25(5):477-488.

M. Hecker, et al. (1993). 'Vasoconstriction and Increased Flow - 2 Principal Mechanisms of Shear Stress-dependent Endothelial Autacoid Release'. Am $J$ Physiol 265(3):H828-H833.

D. Henrion (2005). 'Pressure and flow-dependent tone in resistance arteries Role of myogenic tone'. Archives Des Maladies Du Coeur Et Des Vaisseaux 98(9):913-921.

G. Himpel, et al. (2005). 'Computational Modelling of Isotropic Multiplicative Growth'. CMES 8(2):119-134. 
D. J. S. Hulmes, et al. (1995). 'Radial Packing, Order, and Disorder In Collagen Fibrils'. Biophys J 68(5):1661-1670.

J. Humphrey \& K. Rajagopal (2003). 'A constrained mixture model for arterial adaptations to a sustained step change in blood flow'. Biomech Model Mechan V2(2):109-126.

J. D. Humphrey \& K. R. Rajagopal (2002). 'A constrained mixture model for growth and remodeling of soft tissues'. Math Models Methods Appl Sci 12(3):407-430.

E. Javierre, et al. (2009). 'A mathematical analysis of physiological and morphological aspects of wound closure'. Journal of Mathematical Biology 59(5):605-630.

G. W. Jones \& S. J. Chapman (2012). 'Modeling Growth in Biological Materials'. Siam Review 54(1):52-118.

D. J. Kelly \& P. J. Prendergast (2005). 'Mechano-regulation of stem cell differentiation and tissue regeneration in osteochondral defects'. Journal of Biomechanics 38(7):1413-1422.

S. M. Klisch, et al. (2005). 'A cartilage growth mixture model for infinitesimal strains: solutions of boundary-value problems related to in vitro growth experiments'. Biomech Model Mechan 3(4):209-223.

O. Kratky \& G. Porod (1949). 'Rontgenuntersuchung Geloster Fadenmolekule'. Recl Trav Cnim Pay B 68(12):1106-1122.

E. Kuhl, et al. (2005). 'Remodeling of biological tissue: Mechanically induced reorientation of a transversely isotropic chain network'. J Mech Phys Solids 53(7):1552-1573.

E. Kuhl, et al. (2007). 'Computational modeling of arterial wall growth'. Biomech Model Mechan 6(5):321-331.

E. Kuhl, et al. (2003). 'Computational modeling of growth'. Comput Mech V32(1):71-88.

E. Kuhl \& E. Ramm (2000). 'Microplane modelling of cohesive frictional materials'. Eur J Mech A/Solids 19, special issue:S121-S143.

E. Kuhl \& P. Steinmann (2003). 'Theory and numerics of geometrically nonlinear open system mechanics'. Int J Numer Meth Eng 58(11):1593-1615.

L. Kuo, et al. (1990). 'Coronary Arteriolar Myogenic Response Is Independent of Endothelium'. Circ Res 66(3):860-866.

C. Laviades, et al. (1998). 'Abnormalities of the extracellular degradation of collagen type I in essential hypertension'. Circulation 98(6):535-540.

Y. S. J. Li, et al. (2005). 'Molecular basis of the effects of shear stress on vascular endothelial cells'. J Biomech 38(10):1949-1971.

J. E. Marsden \& T. J. R. Hughes (1994). Mathematical Foundations of Elasticity. Dover Publications.

T. Masaki (1993). 'Endothelins - Homeostatic and Compensatory Actions In the Circulatory and Endocrine Systems'. Endocrine Reviews 14(3):256-268.

T. Masaki, et al. (1991). 'Molecular and Cellular Mechanism of Endothelin Regulation - Implications For Vascular Function'. Circulation 84(4):14571468.

A. Menzel (2004). 'Modelling of anisotropic growth in biological tissues'. Biomech Model Mechan 3(3):147-171. 
A. Menzel (2007). 'A fibre reorientation model for orthotropic multiplicative growth'. Biomech Model Mechan 6:303-320.

C. Miehe, et al. (2004). 'A micro-macro approach to rubber-like materialsPart I: the non-affine micro-sphere model of rubber elasticity'. J Mech Phys Solids 52(11):2617-2660.

M. J. Mulvany \& C. Aalkjaer (1990). 'Structure and Function of Small Arteries'. Physiol Rev 70(4):921-961.

S. I. Murtada, et al. (2010). 'A calcium-driven mechanochemical model for prediction of force generation in smooth muscle'. Biomech Model Mechan 9(6):749-762.

C. J. O'Callaghan \& B. Williams (2000). 'Mechanical strain-induced extracellular matrix production by human vascular smooth muscle cells - Role of TGF-beta 1'. Hypertension 36(3):319-324.

J. P. R. O. Orgel, et al. (2011). 'Molecular and structural mapping of collagen fibril interactions'. Connect Tissue Res 52(1):2-17.

J. P. R. O. Orgel, et al. (2006). 'Microfibrillar structure of type I collagen in situ'. P Natl Acad Sci USA 103(24):9001-9005.

J. P. R. O. Orgel, et al. (2001). 'The in situ supermolecular structure of type I collagen'. Structure 9(11):1061-1069.

G. Osol (1995). 'Mechanotransduction By Vascular Smooth-muscle'. J Vasc Res 32(5):275-292.

G. K. Owens (1989). 'Control of Hypertrophic Versus Hyperplastic Growth of Vascular Smooth-muscle Cells'. Am J Physiol 257(6):H1755-H1765.

G. K. Owens (1995). 'Regulation of Differentiation of Vascular Smooth-muscle Cells'. Physiol Rev 75(3):487-517.

G. K. Owens, et al. (1981). 'Smooth-muscle Cell Hypertrophy Versus Hyperplasia In Hypertension'. P Natl Acad Sci-biol 78(12):7759-7763.

H. Pang, et al. (2011). 'Computational modeling of bone density profiles in response to gait: A subject specific approach.'. Biomech Model Mechan In press:doi:10.1007/s10237-011-0318-y.

E. Porreca, et al. (1997). 'Increased transforming growth factor-beta production and gene expression by peripheral blood monocytes of hypertensive patients'. Hypertension 30(1):134-139.

E. W. Raines (2004). 'PDGF and cardiovascular disease'. Cytokine Growth F $R$ 15(4):237-254.

E. K. Rodriguez, et al. (1994). 'Stress-dependent finite growth in soft elastic tissues'. J Biomech 27(4):455-467.

G. M. Rubanyi \& M. A. Polokoff (1994). 'Endothelins - Molecular-biology, Biochemistry, Pharmacology, Physiology, and Pathophysiology'. Pharmacol Rev 46(3):325-415.

R. Sarzani, et al. (1989). 'Growth-factor Expression In Aorta of Normotensive and Hypertensive Rats'. J Clin Invest 83(4):1404-1408.

B. D. Schaan, et al. (2007). 'Correction:' Serum transforming growth factor beta-1 (TGF-beta-1) levels in diabetic patients are not associated with preexistent coronary artery disease'. Cardiovasc Diabetol 6:19.

A. J. Schriefl, et al. (2012). 'Determination of the layer-specific distributed collagen fiber orientations in human thoracic and abdominal aortas and common iliac arteries'. J Roy Soc Interface In Press. 
R. Schubert \& M. J. Mulvany (1999). 'The myogenic response: established facts and attractive hypotheses'. Clinical Science 96(4):313-326.

Z. L. Shen, et al. (2008). 'Stress-strain experiments on individual collagen fibrils'. Biophysical Journal 95(8):3956-3963.

R. Skalak, et al. (1982). 'Analytical description of growth'. J Theor Biol 94(3):555-577.

L. A. Taber (1995). 'Biomechanics of Growth, Remodeling, and Morphogenesis'. Appl. Mech. Rev. 48:487-545.

C. Truesdell \& W. Noll (2004). The Non-Linear Field Theories of Mechanics. Springer-Verlag, 3rd edn.

M. C. H. van der Meulen \& P. J. Prendergast (2000). 'Mechanics in skeletal development, adaptation and disease RID D-1549-2010'. Philosophical Transactions of the Royal Society of London Series A-mathematical Physical and Engineering Sciences 358(1766):565-578.

J. A. J. van der Rijt, et al. (2006). 'Micromechanical testing of individual collagen fibrils'. Macromolecular Bioscience 6(9):697-702.

T. Waffenschmidt, et al. (2011). 'Anisotropic density growth of bone - a computational microsphere approach' Submitted for publication.

J. L. Wrana, et al. (1994). 'Mechanism of Activation of the Tgf-beta Receptor'. Nature 370(6488):341-347.

C. C. Wu \& D. F. Bohr (1990). 'Role of Endothelium In the Response To Endothelin In Hypertension'. Hypertension 16(6):677-681.

M. A. Zulliger, et al. (2004). 'A constitutive formulation of arterial mechanics including vascular smooth muscle tone'. Am J Physiol Heart Circ Physiol 287(3):H1335-1343. 